\title{
2. FORAMINIFERAL RANGE CHARTS FOR ARABIAN SEA AND RED SEA SITES, LEG 23
}

W. H. Akers, Chevron Oil Company, New Orleans, Louisiana

The total abundance data is a visual estimate of the numbers of planktonic foraminifera in a strew on a 60 hole, 28 ply slide, 149 micron (100 mesh) screen size. Standard sample size for shore-lab work is $2 \mathrm{cc}$. It is intended as an approximate indication of whether a particular sample (level) is rich or poor in planktonic foraminifera. The following numerical scheme has been adopted for expressing this abundance:

$$
\begin{array}{lll}
\text { 0: } & \text { Absent } & \\
\text { 1: } & <10^{1} & \text { specimens } \\
\text { 2: } & 10^{1}-10^{2} & \text { specimens } \\
\text { 3: } & 10^{2}-10^{3} & \text { specimens } \\
\text { 4: } & 10^{3}-10^{4} & \text { specimens } \\
\text { 5: } & >10^{4} & \text { specimens }
\end{array}
$$

The relative abundance of each taxon in a strew on a 60

\begin{tabular}{|c|c|}
\hline Relative Abundance & No. Specimens \\
\hline \multicolumn{2}{|l|}{$0=$ Absent } \\
\hline $1=$ Rare & $1-10$ \\
\hline $2=$ Moderately Rare & $11-25$ \\
\hline $3=$ Common & $26-50$ \\
\hline $4=$ Abundant & $51-100$ \\
\hline $5=$ Very Abundant & $>100$ \\
\hline $\begin{aligned} & *= \text { Hundreds to } \\
& \text { Thousands of } \\
& \text { Specimens }\end{aligned}$ & \\
\hline
\end{tabular}
hole, 28 ply slide, 0.149 micron (100 mesh) screen size, is shown by a number as well as the state of preservation by a letter. If preservation is good no letter notation is made. The following scheme has been adopted for expressing relative abundance and preservation:
Preservation

$\mathrm{D}=$ Dissolution

$\mathrm{M}=$ Mechanical

Erosion

$\mathrm{R}=$ Reworked 


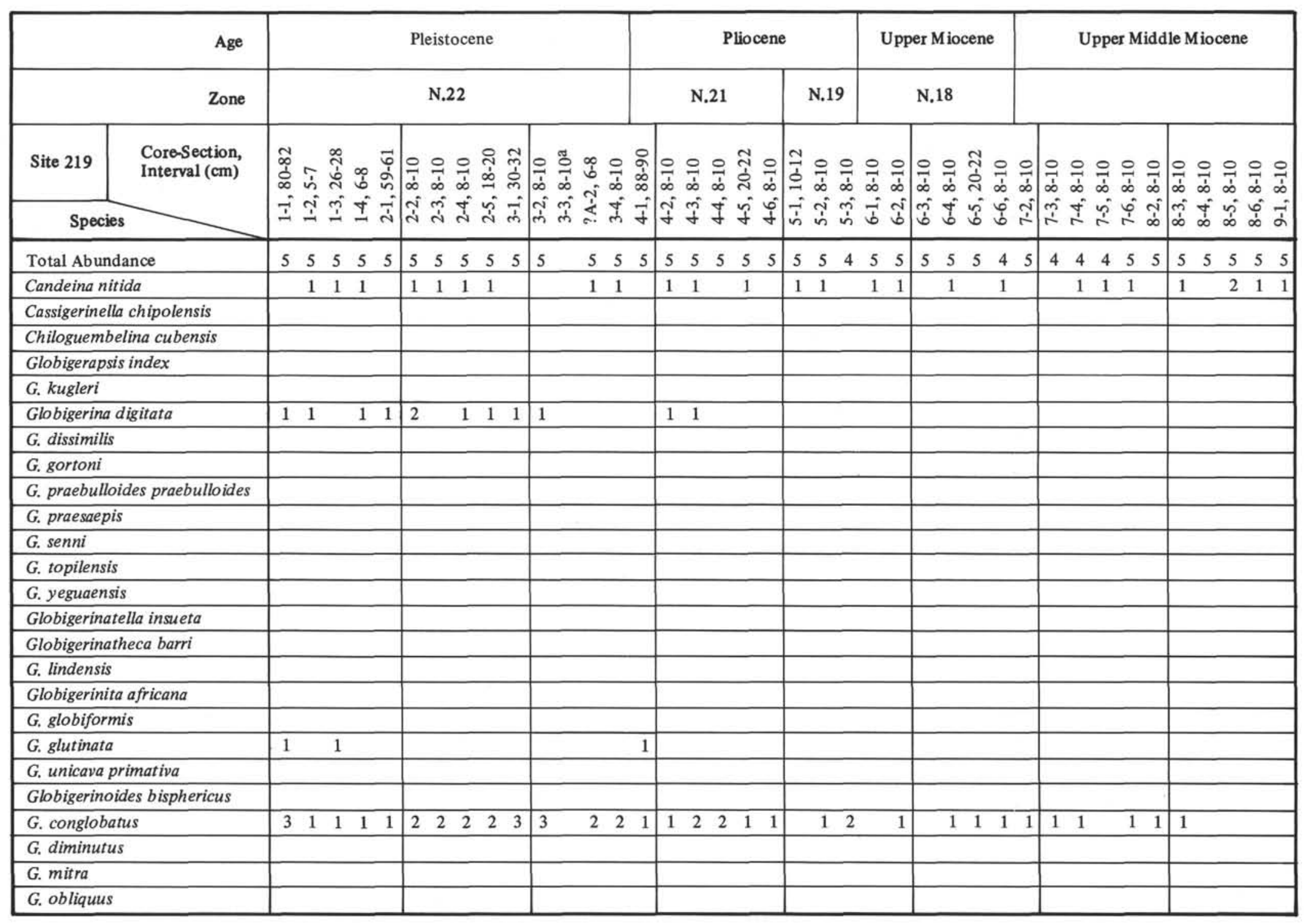




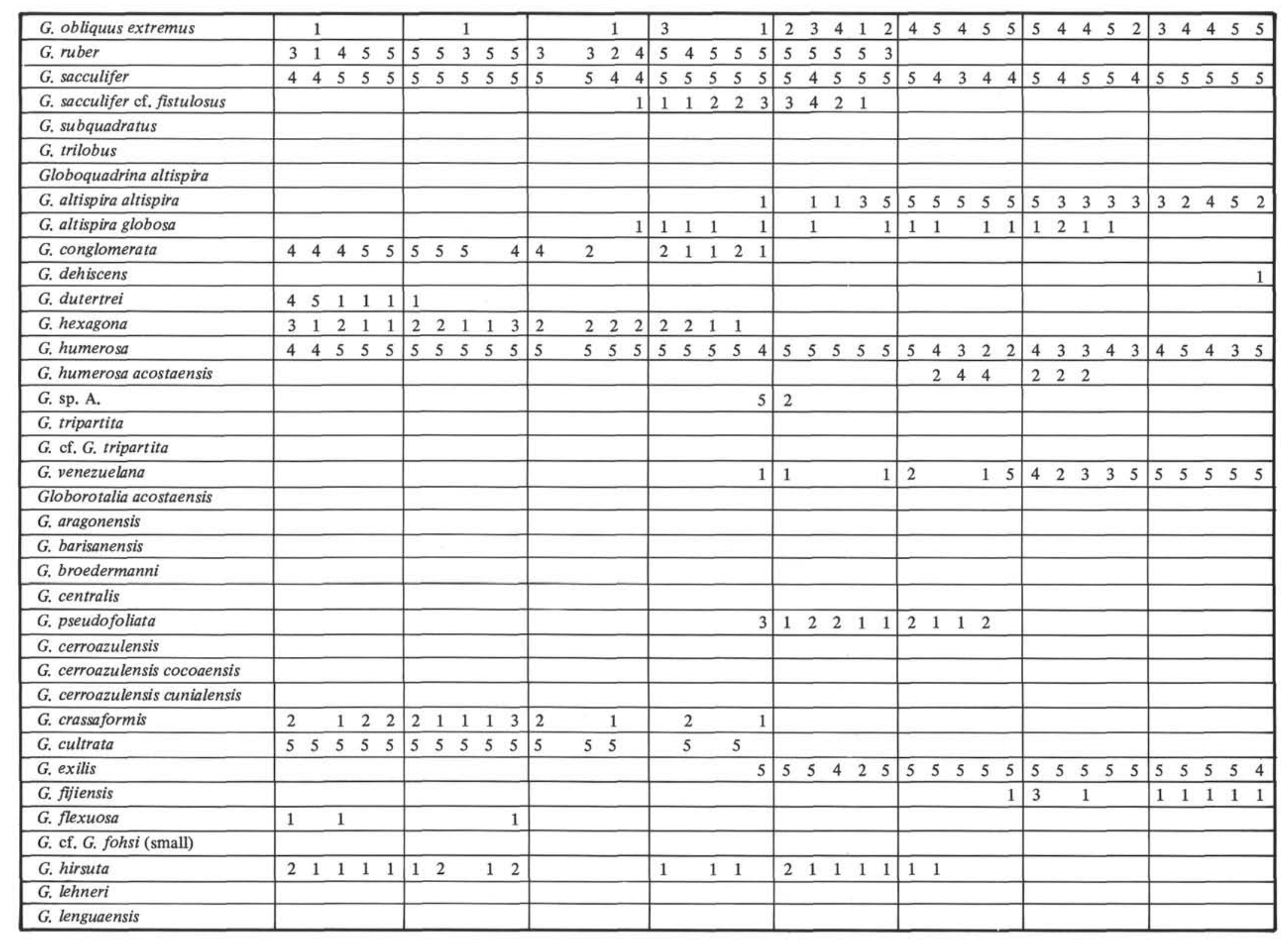




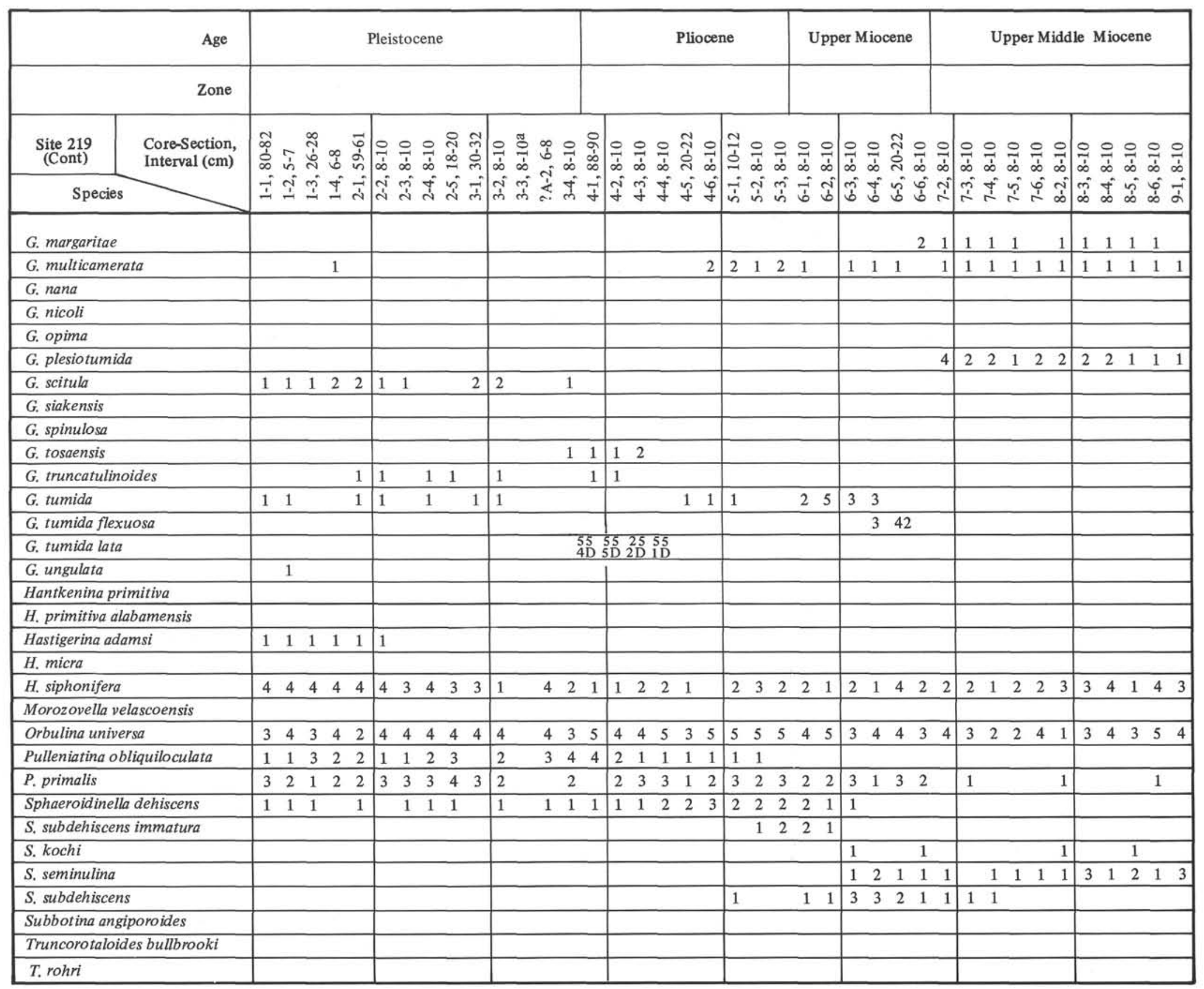




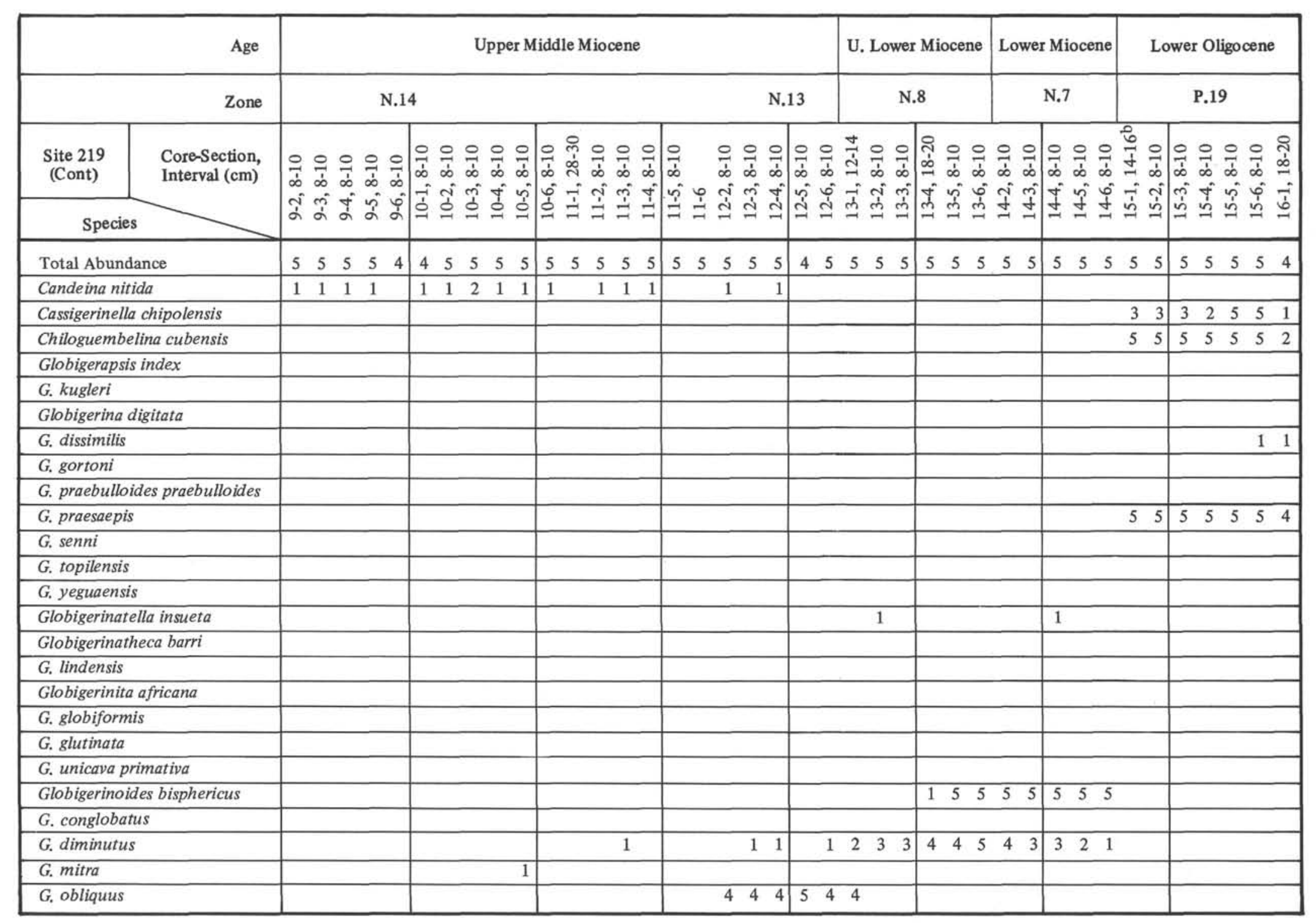




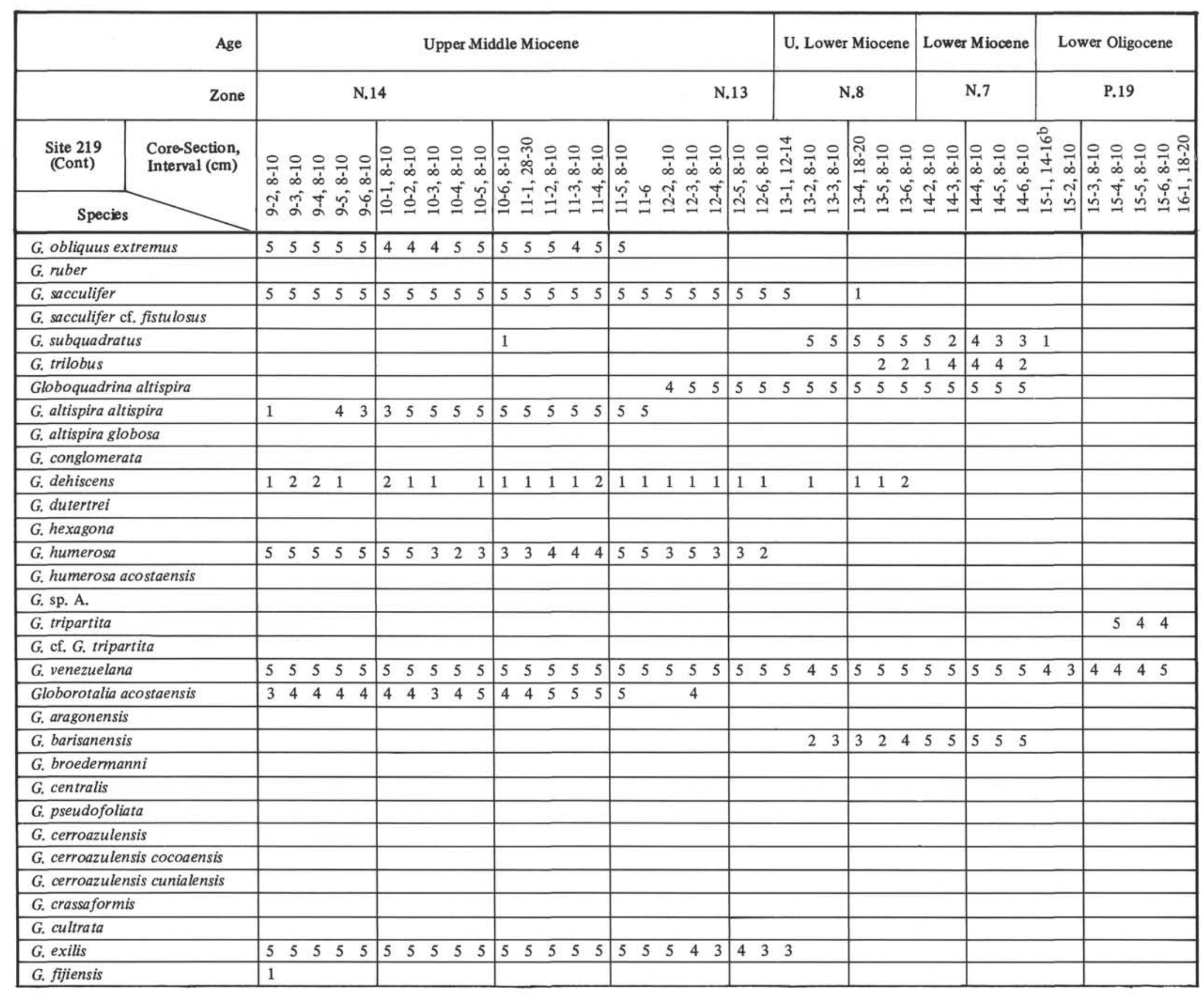




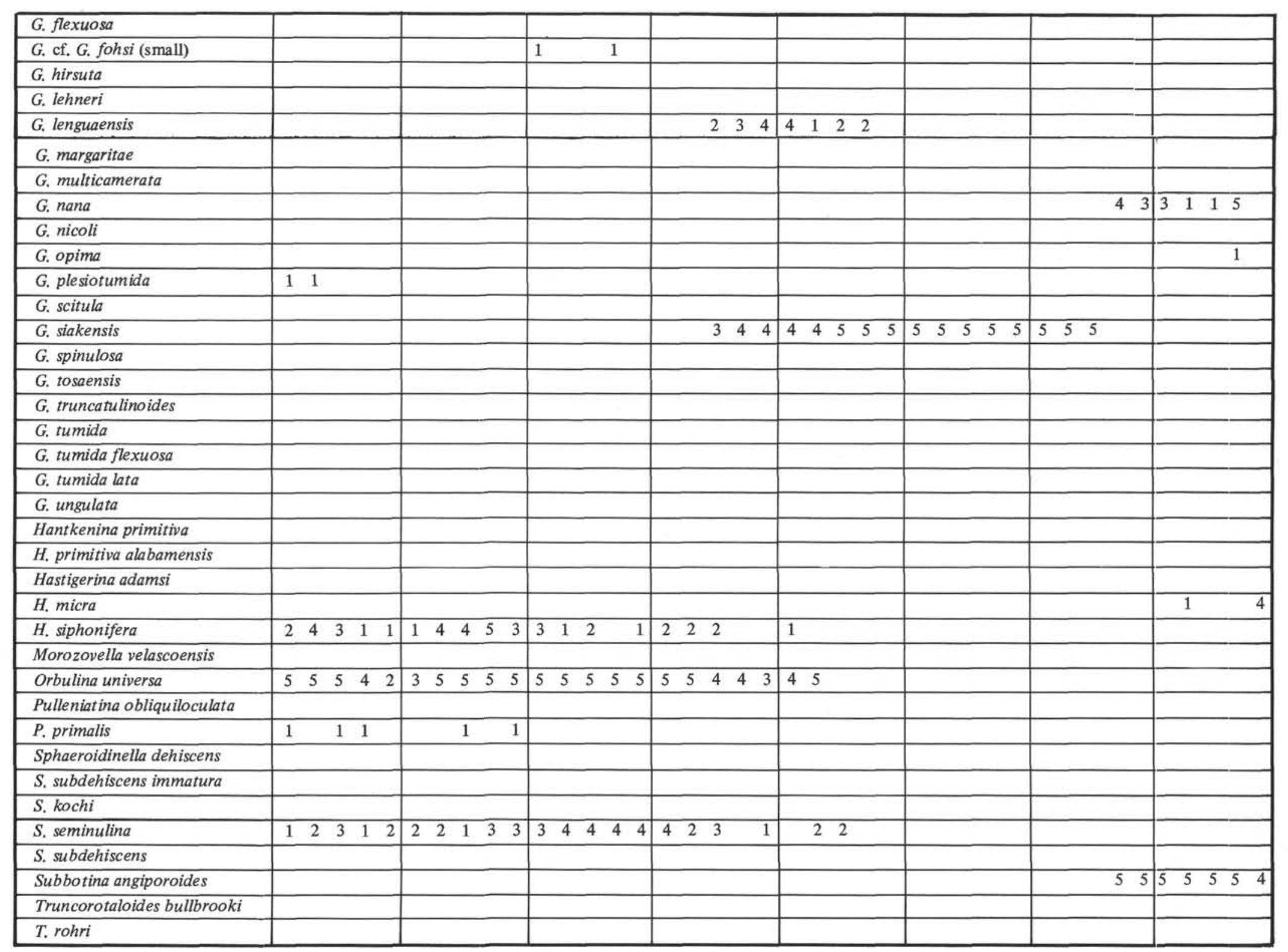




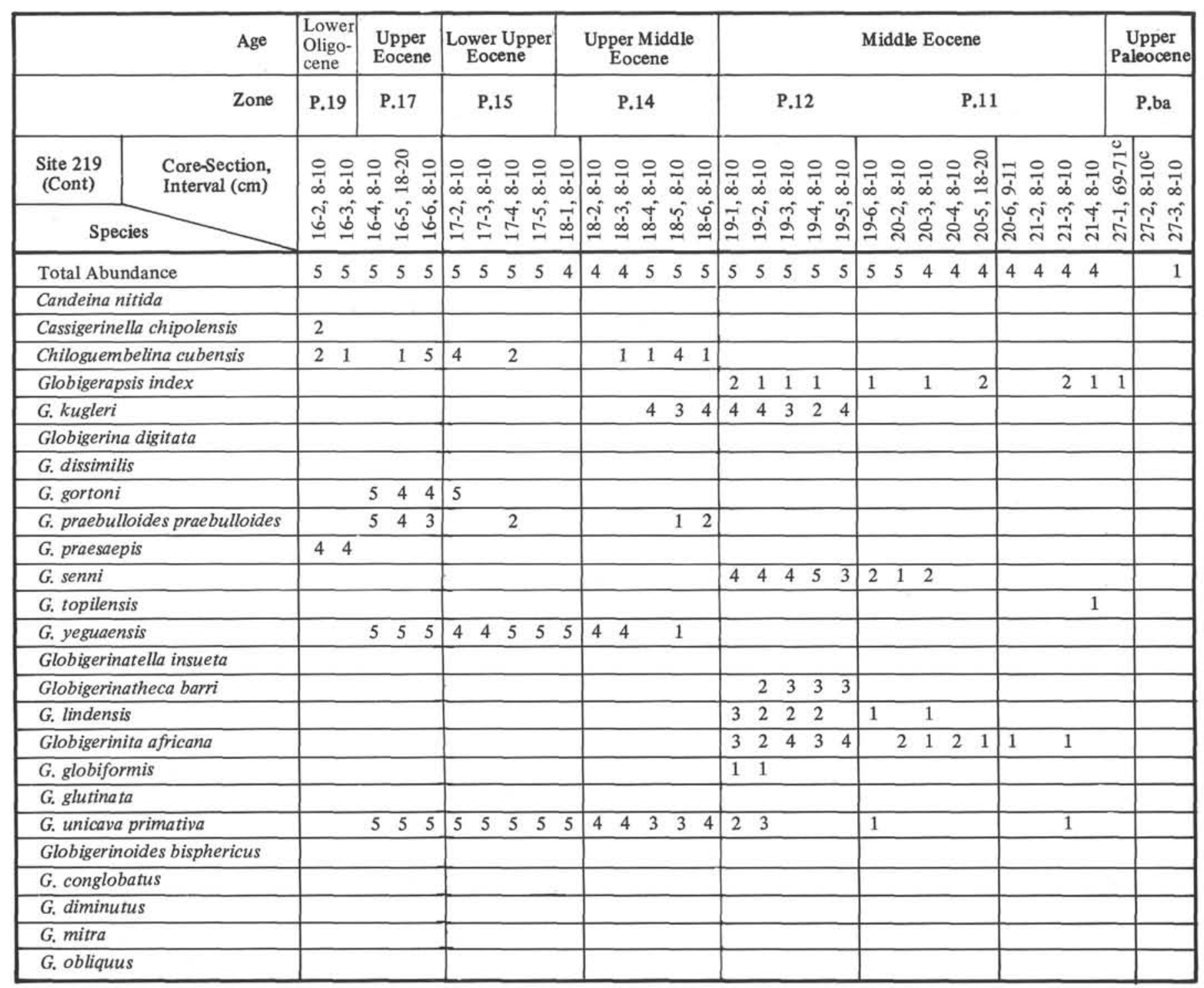




\begin{tabular}{|c|c|c|c|c|c|c|c|}
\hline G. obliquus extremus & & & & & & & \\
\hline G. ruber & & & & & & & \\
\hline G. sacculifer & & & & & & & \\
\hline G. sacculifer cf. fistulosus & & & & & 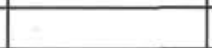 & & \\
\hline G. subquadratus & & & & & & & \\
\hline G. trilobus & & & & & & & \\
\hline Globoquadrina altispira & & & & & & & \\
\hline G. altispira altispira & & & & & & & \\
\hline G. altispira globosa & & & & & & & \\
\hline G. conglomerata & & & & & & & \\
\hline G. dehiscens & & & & & & & \\
\hline G. dutertrei & & & & & & & \\
\hline G. hexagona & & & & & & & \\
\hline G. humerosa & & & & & & & \\
\hline G. humerosa acostaensis & & & & & & & \\
\hline G. sp. A. & & & & & & & \\
\hline G. tripartita & 2 & & 1 & & & & \\
\hline G. cf. G. tripartita & $\begin{array}{lll}5 & 4 & 3 \\
\end{array}$ & $\begin{array}{lll}4 & 5 & 3\end{array}$ & & & & & \\
\hline G. venezuelana & 1 & & & & & & \\
\hline Globorotalia acostaensis & & & & & & & \\
\hline G. aragonensis & & & & & \begin{tabular}{lll|}
2 & 3 & 4 \\
\end{tabular} & $\begin{array}{lll}4 & 2 & 2\end{array}$ & \\
\hline G. barisanensis & & & & & 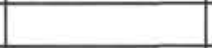 & & \\
\hline G. broedermanni & & & & & \begin{tabular}{lll|}
1 & 3 & 4 \\
\end{tabular} & $\begin{array}{lll}3 & 5 & 5\end{array}$ & \\
\hline G. centralis & & & 125 & $\begin{array}{|lllll|}5 & 5 & 5 & 4 & 5\end{array}$ & $\begin{array}{|lllll|}4 & 5 & 1 & 1 & 2\end{array}$ & $\begin{array}{lll}4 & 1 & 1\end{array}$ & \\
\hline G. pseudofoliata & & & & 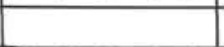 & & & \\
\hline G. cerroazulensis & & & & $\begin{array}{|lllll|}5 & 4 & 1 & 3 & 5\end{array}$ & 32 & & \\
\hline G. cerroazulensis cocoaensis & $\begin{array}{lll}5 & 4 & 4\end{array}$ & $\begin{array}{llll}5 & 4 & 3 & 1\end{array}$ & $\begin{array}{lllll}2 & 4 & 1 & 1 & 4\end{array}$ & & & & \\
\hline G. cerroazulensis cunialensis & $\begin{array}{lll}3 & 1 & 1\end{array}$ & 1 & 1 & & & & \\
\hline G. crassaformis & & & & & & & \\
\hline G. cultrata & & & & & & & \\
\hline G. exilis & & & & & & & \\
\hline G. fijiensis & & & & & & & \\
\hline G. flexuosa & & & & & & & \\
\hline G. cf. G. fohsi (small) & & & & & & & \\
\hline G. hirsuta & & & & & & & \\
\hline G. lehneri & & & 235 & & $\begin{array}{llll}3 & 2 & 2 & 4 \\
\end{array}$ & 12 & \\
\hline G. lenguaensis & & & & & & & \\
\hline
\end{tabular}




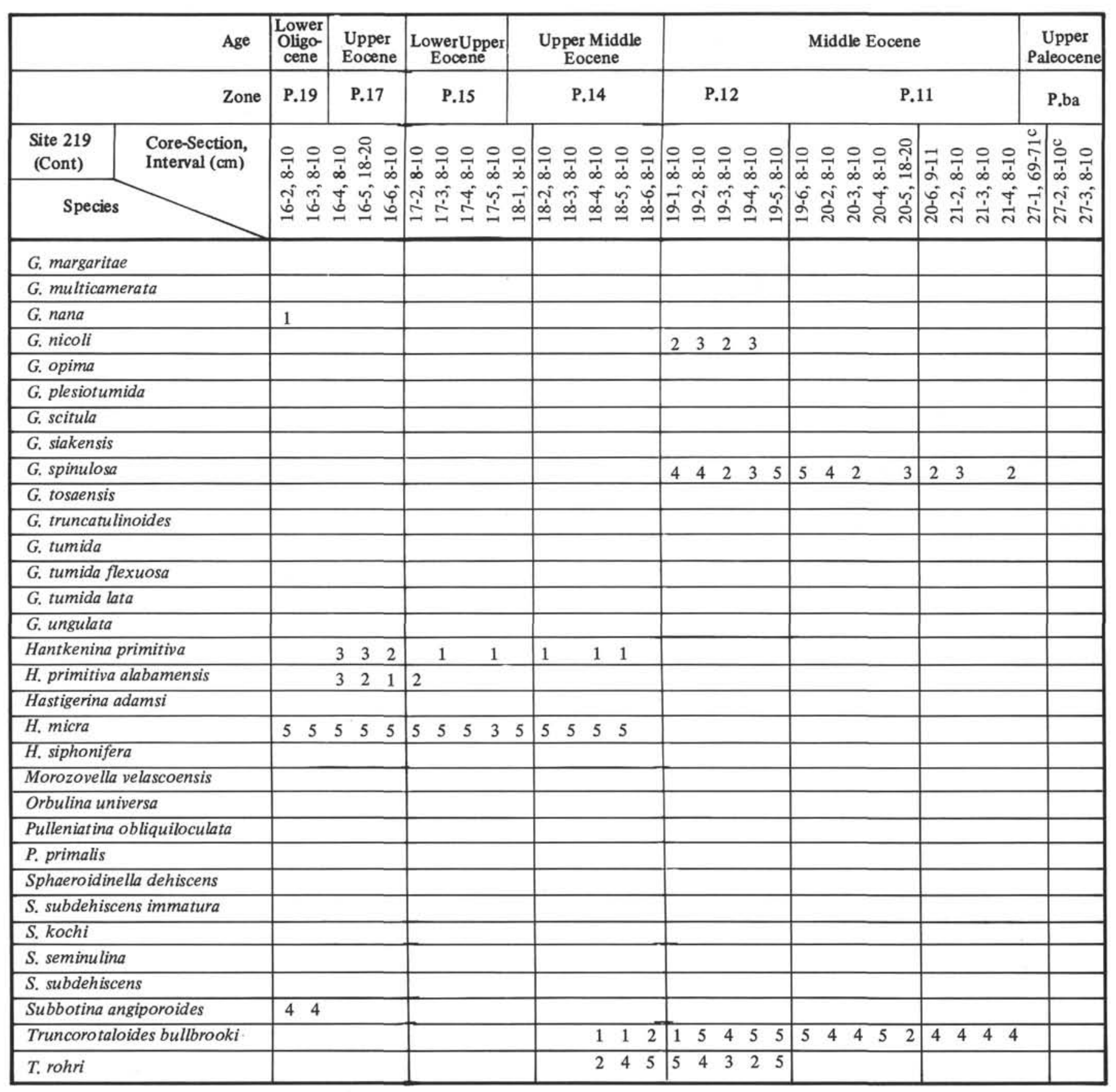




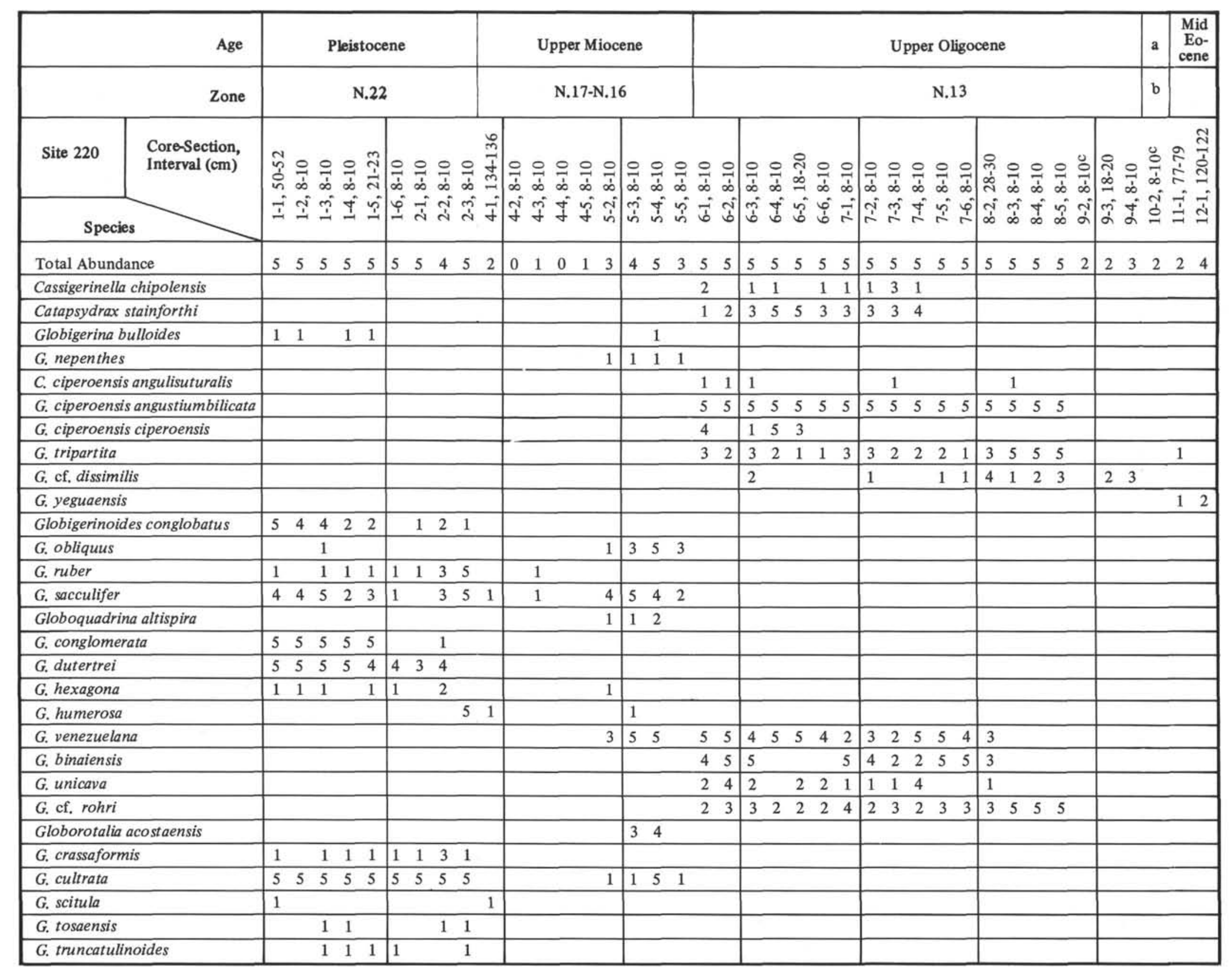




\begin{tabular}{|c|c|c|c|c|c|c|c|c|c|c|c|c|c|c|c|c|}
\hline & Age & & & leistoc & & & & Upper Mioce & & & & & Upper Oligoc & cene & a & \begin{tabular}{|c} 
Mid \\
Eo \\
cene
\end{tabular} \\
\hline & Zone & & & $\mathrm{N} .2$ & & & & N.17-N.16 & & & & & $\mathrm{N} .13$ & & b & \\
\hline Site 220 & $\begin{array}{l}\text { Core-Section, } \\
\text { Interval }(\mathrm{cm})\end{array}$ & 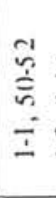 & & 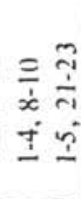 & & 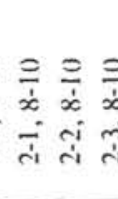 & 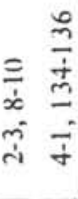 & 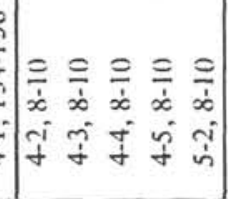 & & $\begin{array}{l}= \\
\dot{x} \\
\dot{y} \\
\dot{n} \\
\vdots\end{array}$ & 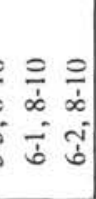 & 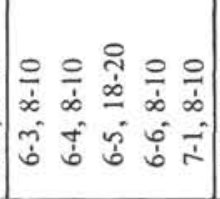 & 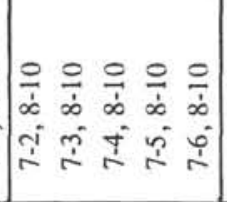 & 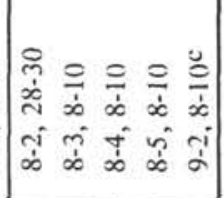 & 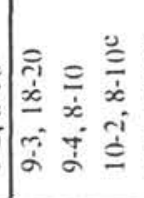 & 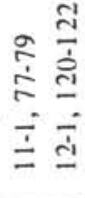 \\
\hline G. tumida & & 5 & $\begin{array}{ll}5 & 4 \\
\end{array}$ & 25 & \begin{tabular}{|l|l}
5 & 5 \\
\end{tabular} & $5 \quad 5$ & 1 & & & & & & & & & \\
\hline G. opima $n$ & & & & & & & & & & & 22 & $\begin{array}{|lll|}2 & 1 & 1 \\
\end{array}$ & \begin{tabular}{lllll|}
1 & 1 & 1 & 2 & 1
\end{tabular} & $\begin{array}{ll}1 & 1 \\
\end{array}$ & 1 & \\
\hline G. siakensi. & & & & & & & & & & & $\begin{array}{|ll|}5 & 5 \\
\end{array}$ & $\begin{array}{|lllll|}5 & 5 & 5 & 5 & 5 \\
\end{array}$ & $\begin{array}{|lllll|}5 & 5 & 5 & 5 & 5 \\
\end{array}$ & $\begin{array}{llll}5 & 4 & 4 & 3 \\
\end{array}$ & & \\
\hline G. aragone, & & & & & & & & & & & & & & & & 3 \\
\hline G. bullbroc & & & & & & & & & & & & & & & & 3 \\
\hline G. spinulos & & & & & & & & & & & & & & & & \\
\hline G. velascoe & & & & & & & & & & & & & & & & \\
\hline "Globorotc & "broedermanni & & & & & & & & & & & & & & & 3 \\
\hline Globorotal & les sp. & & & & & & & & & & \begin{tabular}{ll|}
5 & 5 \\
\end{tabular} & $\begin{array}{|lll|}5 & 5 & 5 \\
\end{array}$ & $\begin{array}{|lllll|}5 & 5 & 5 & 5 & 5 \\
\end{array}$ & $\begin{array}{llll}5 & 5 & 3 & 4 \\
\end{array}$ & & \\
\hline Globigerap. & kugleri & & & & & & & & & & & & & & & 3 \\
\hline Guembelin & ubensis & & & & & & & & & 4 & & & & & & \\
\hline Hastigerina & phonifera & 5 & 15 & 24 & 4 & 23 & & 1 & & 4 & & & & & & \\
\hline Morozovell & ehneri & & & & & & & & & & & & & & & 1 \\
\hline Orbulina $u$ & & 4 & 4 & 33 & 3 & 1 & 1 & 1 & 23 & 3 & & & & & & \\
\hline Pseudohast & rina micra & & & & & & & & & & & & & & & \\
\hline Pulleniatin & bliquiloculata & & 55 & 55 & 5 & 55 & 5 & & & & & & & & & \\
\hline Sphaeroidi & Ila dehiscens & 3 & 32 & 32 & 23 & \begin{tabular}{|ll}
5 & 4 \\
\end{tabular} & & & & & & & & & & \\
\hline Sphaeroidi & lopsis seminulina & & & & & & & 4 & 45 & 5 & & & & & & \\
\hline S. subdehis & & & & & & & 1 & $\begin{array}{ll}1 & 4 \\
\end{array}$ & 4 & 4 & & & & & & \\
\hline Juvenile In & terminants & & & & & & & & & & & & & & & \\
\hline
\end{tabular}




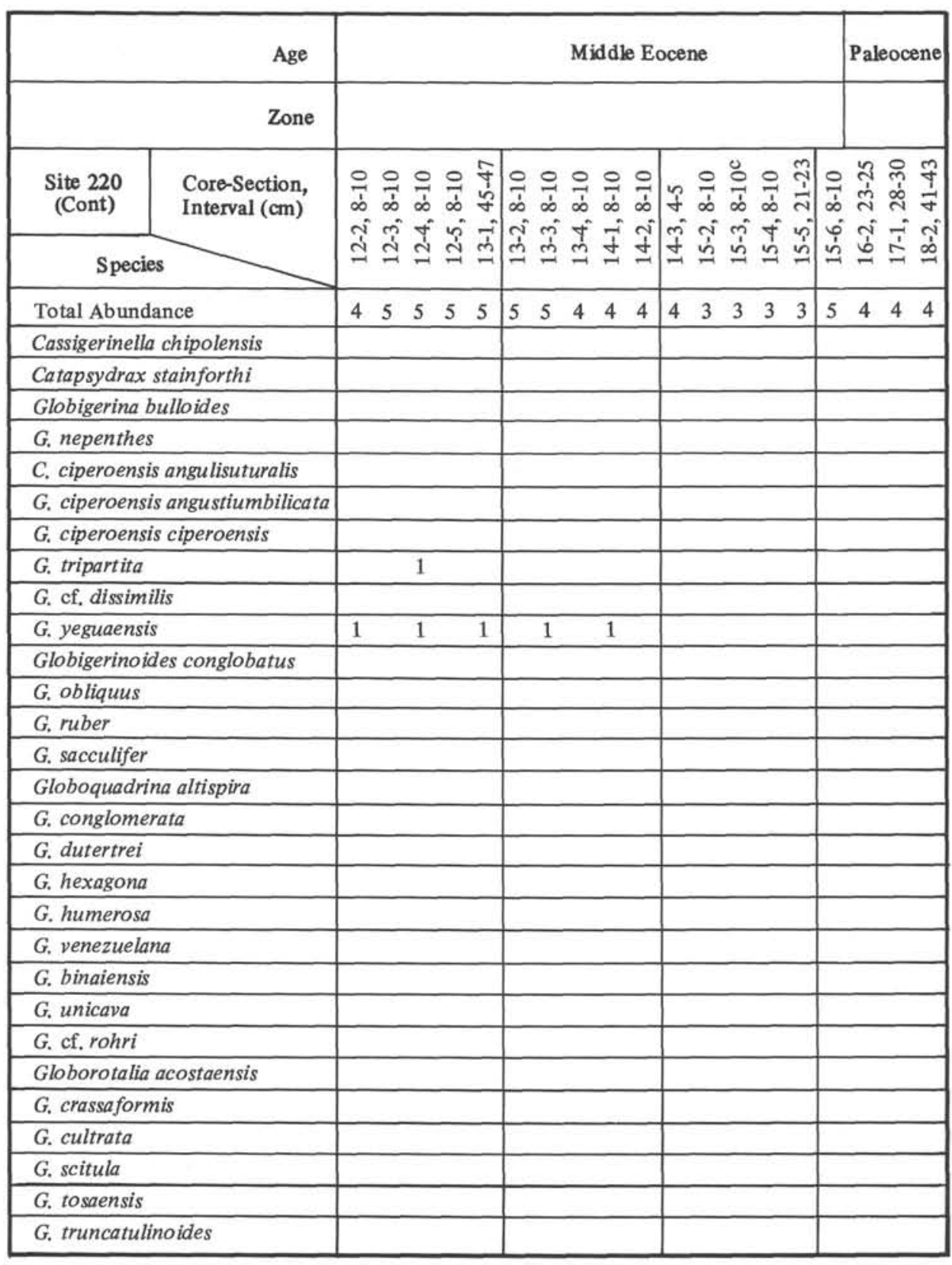




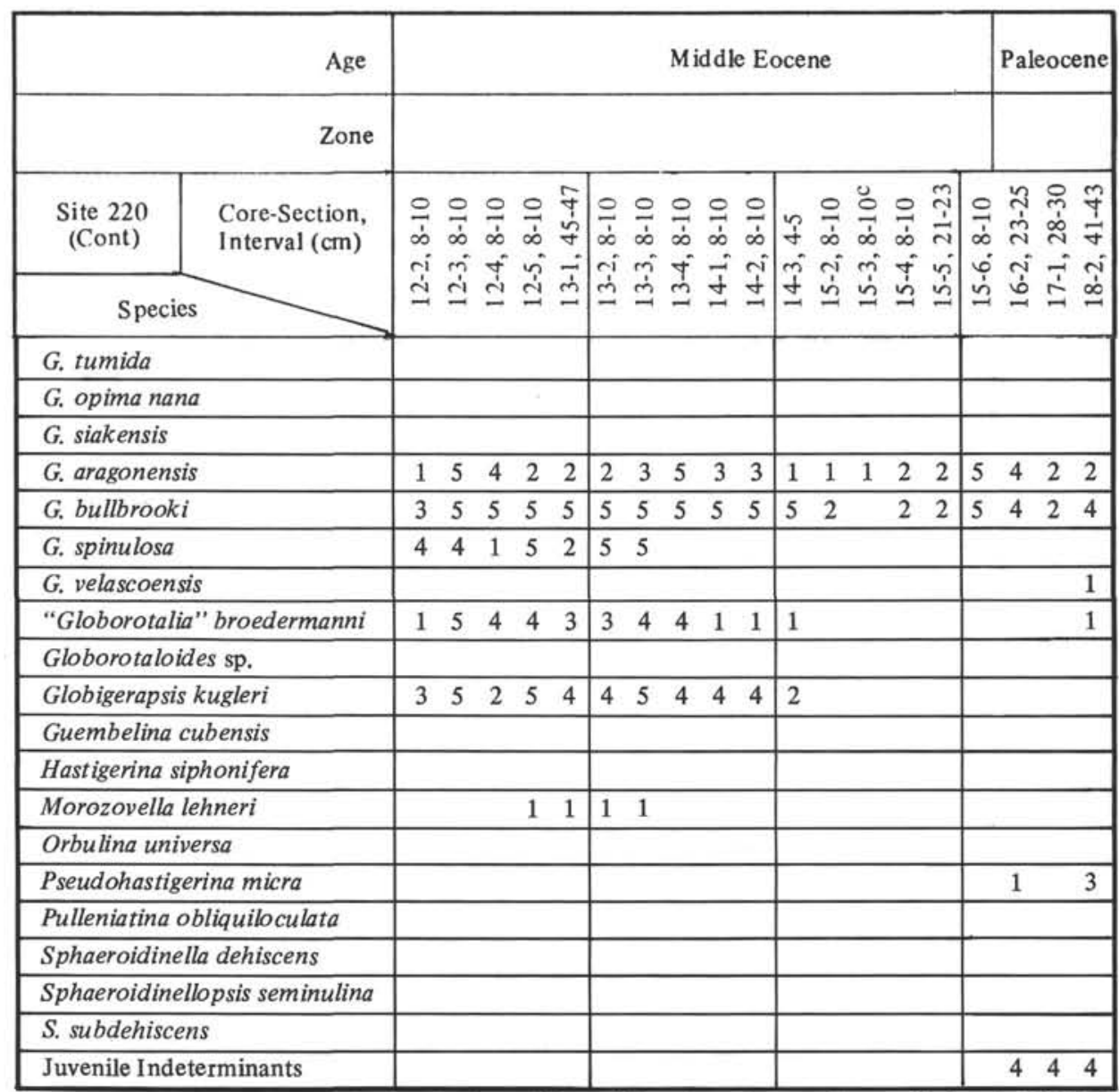

Note: Site 221, in all cores, planktonic foraminifera either absent or nondiagnostic due to solution.

${ }^{\mathrm{a}}$ Lower Oligocene.

b.19 or P.18.

${ }^{\mathrm{c}}$ Indeterminant juveniles. 


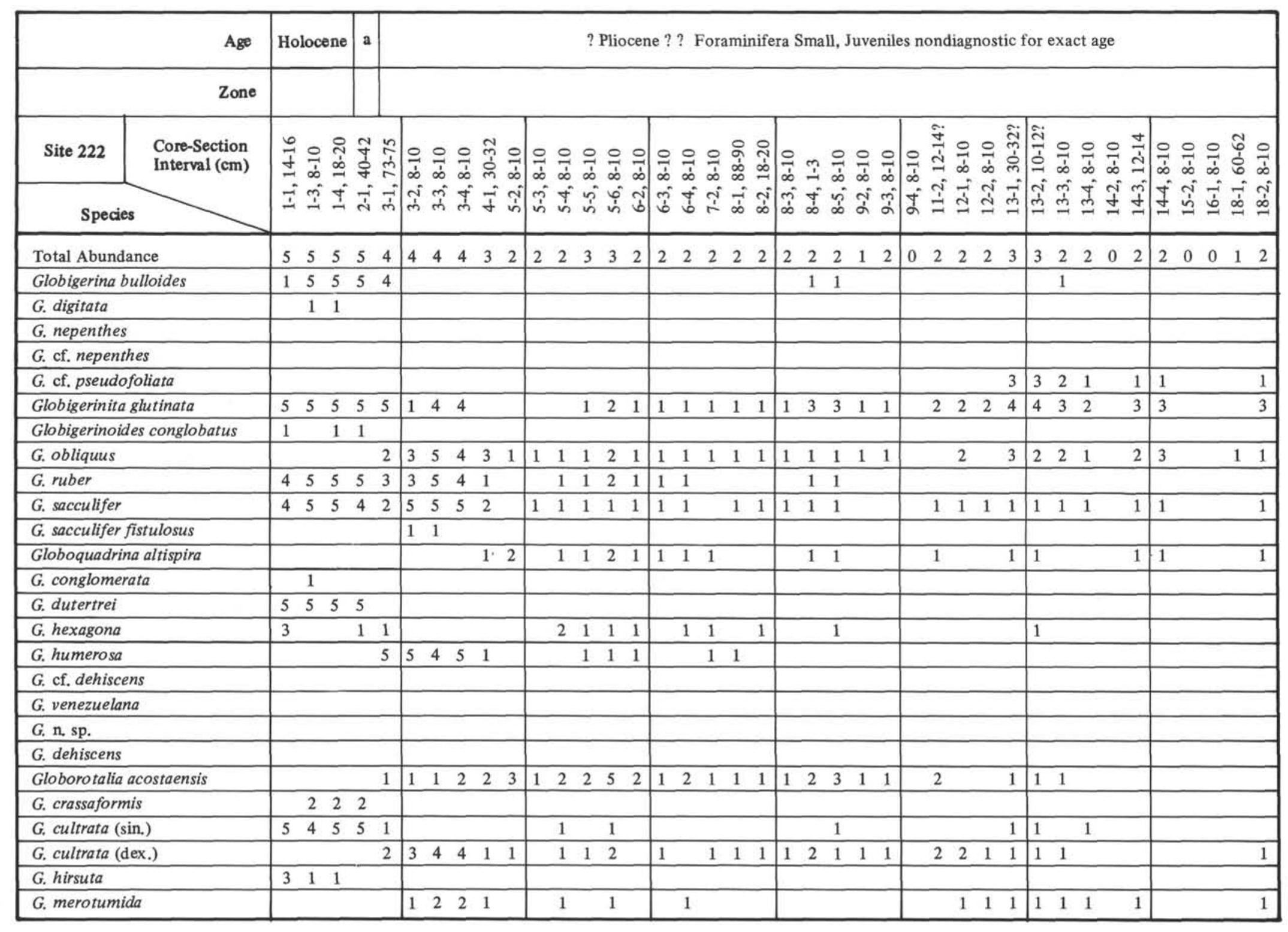




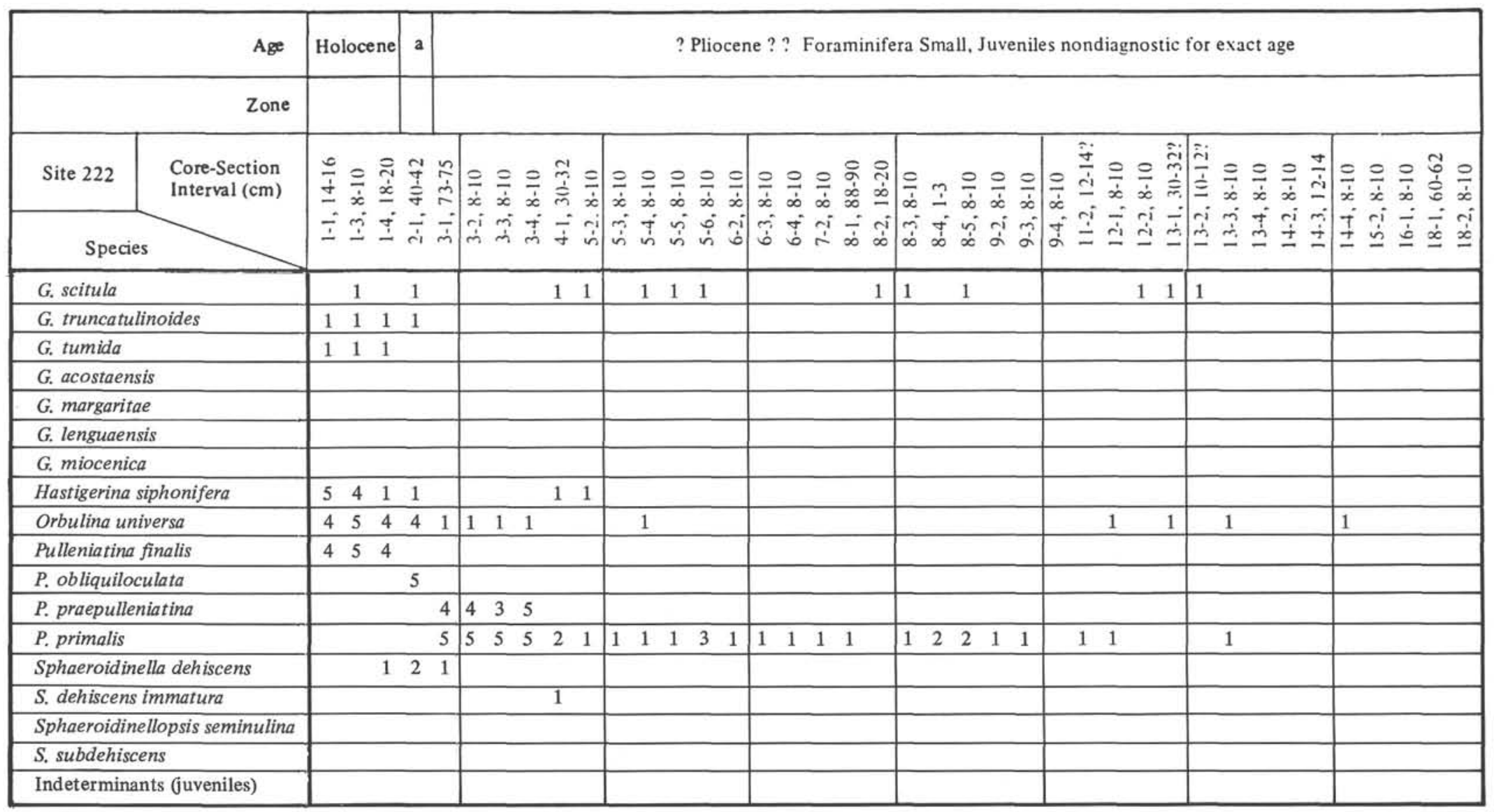




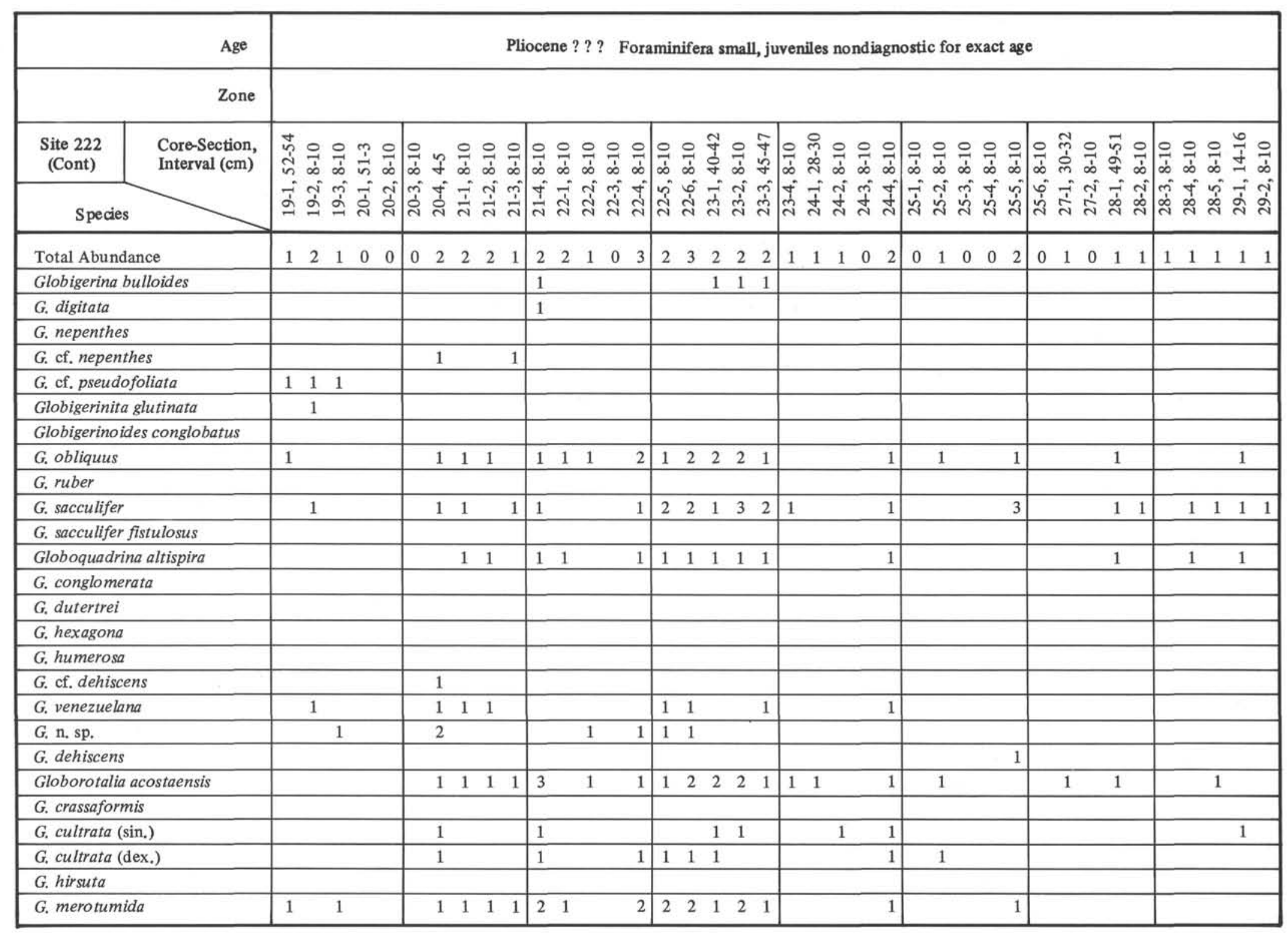




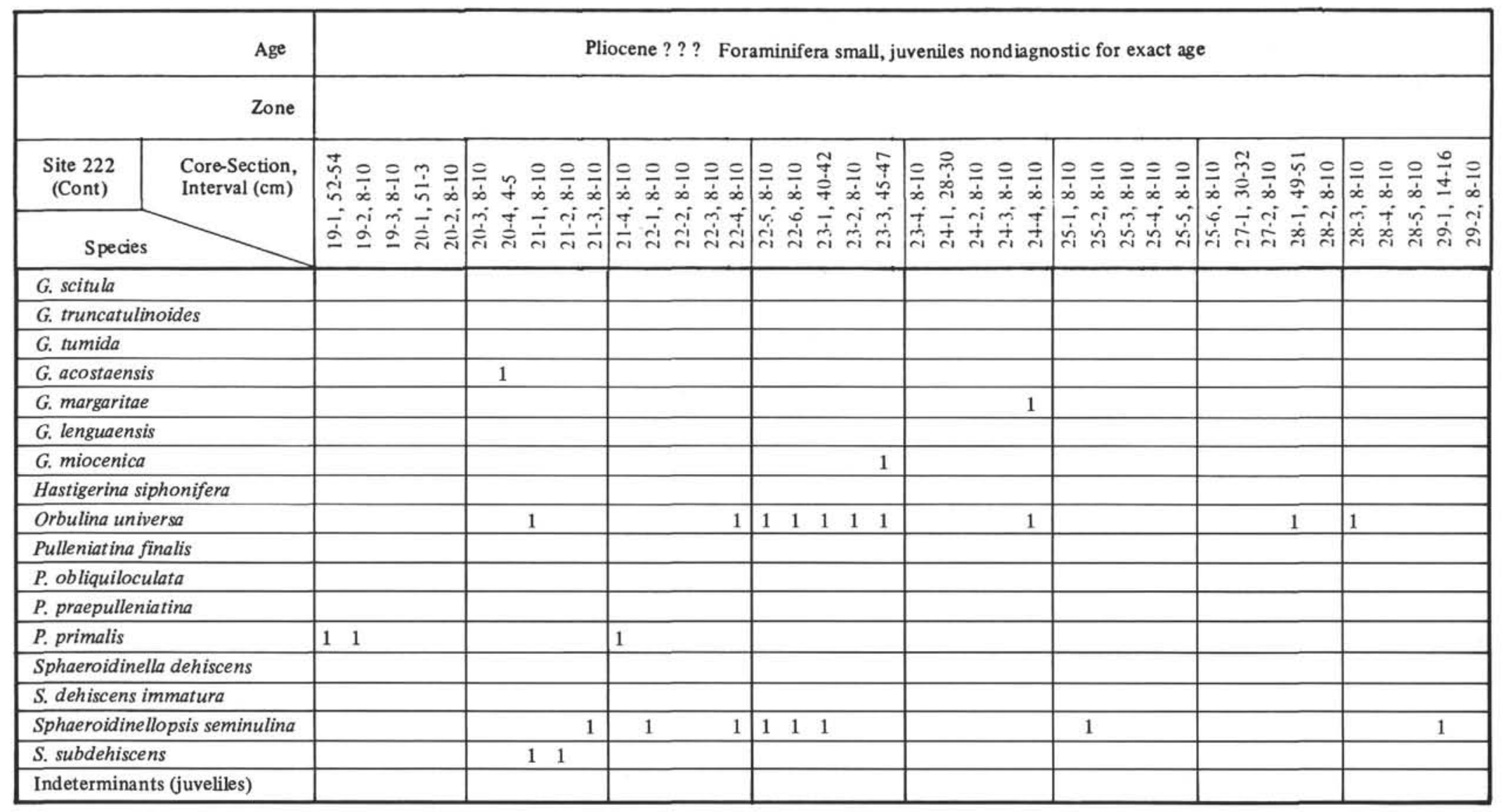




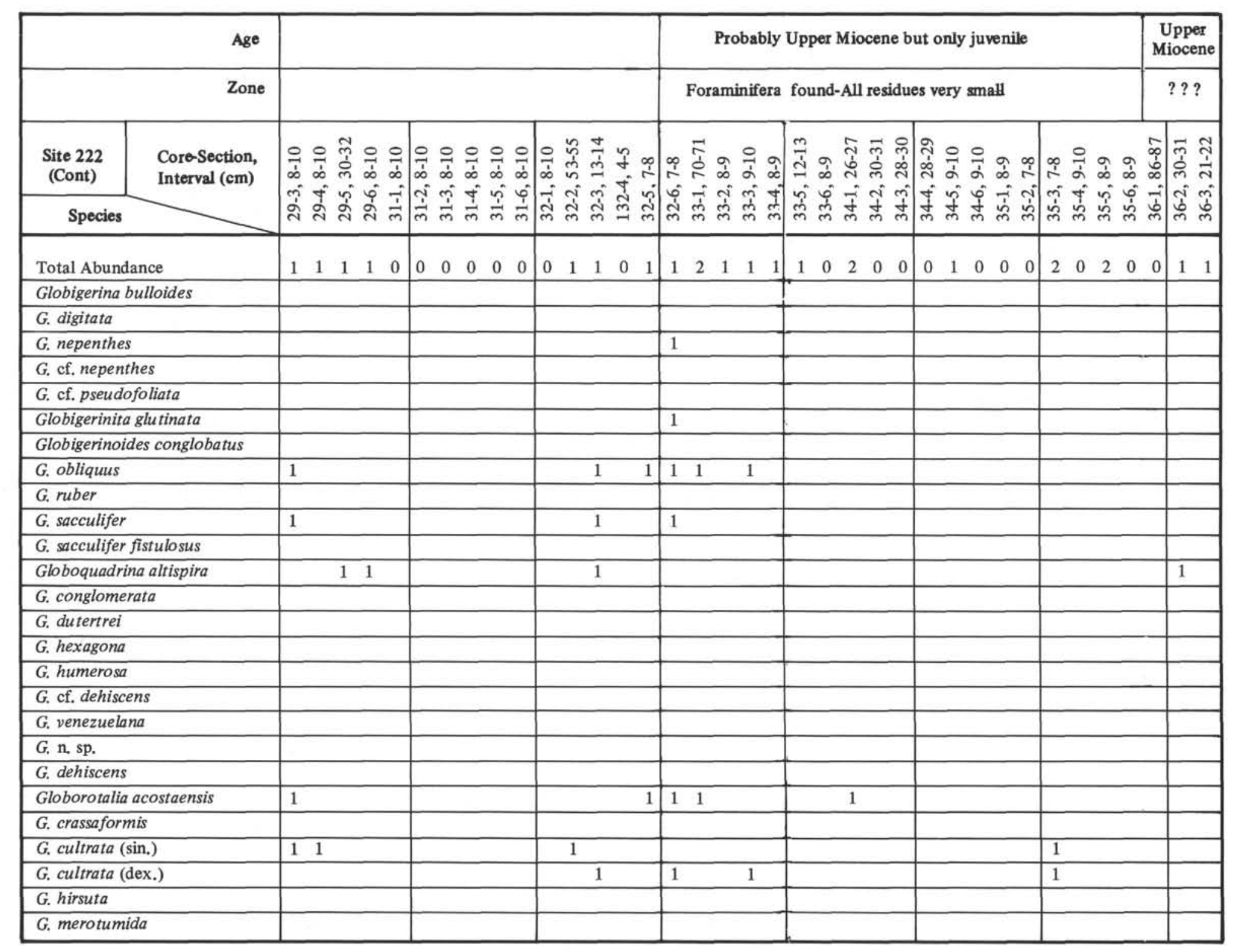




\begin{tabular}{|c|c|c|c|c|c|c|c|c|c|}
\hline & Age & \multicolumn{7}{|c|}{ Probably Upper Miocene but only juvenile } & $\begin{array}{l}\text { Upper } \\
\text { Miocene }\end{array}$ \\
\hline & Zone & \multicolumn{7}{|c|}{ Foraminifera found-All residues very small } & $? ? ?$ \\
\hline $\begin{array}{l}\text { Site } 222 \\
\text { (Cont) }\end{array}$ & $\begin{array}{l}\text { Core-Section, } \\
\text { Interval }(\mathrm{cm})\end{array}$ & 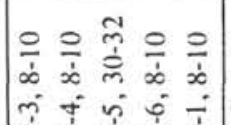 & 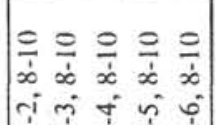 & 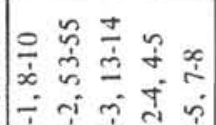 & 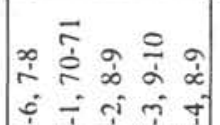 & 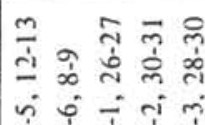 & 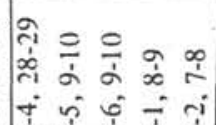 & 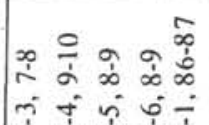 & 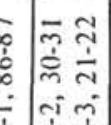 \\
\hline \multicolumn{2}{|c|}{ Species } & & $\bar{m} \bar{m} \bar{m} \bar{m}$ & 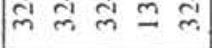 & लिले लm ले & $m m \dot{m} \dot{m}$ & $\dot{m} \dot{m} \dot{m} \dot{m}$ & $\ddot{m} \approx m \tilde{m}$ & $\dot{m}$ \\
\hline \multicolumn{2}{|c|}{ G. scitula } & & & & $\begin{array}{ll}1 & 1 \\
\end{array}$ & & & & \\
\hline \multicolumn{10}{|c|}{ G. truncatulinoides } \\
\hline \multicolumn{10}{|c|}{ G. tumida } \\
\hline \multicolumn{10}{|c|}{ G. acostaensis } \\
\hline \multicolumn{10}{|c|}{ G. margaritae } \\
\hline \multicolumn{10}{|c|}{ G. lenguaensis } \\
\hline G. miocen & & & & & & 1 & & & \\
\hline \multicolumn{10}{|c|}{ Hastigerina siphonifera } \\
\hline \multicolumn{10}{|c|}{ Orbulina universa } \\
\hline \multicolumn{10}{|c|}{ Pulleniatina finalis } \\
\hline \multicolumn{10}{|c|}{ P. obliquiloculata } \\
\hline \multicolumn{10}{|c|}{ P. praepulleniatina } \\
\hline \multicolumn{10}{|c|}{ P. primalis } \\
\hline \multicolumn{10}{|c|}{ Sphaeroidinella dehiscens } \\
\hline \multicolumn{10}{|c|}{ S. dehiscens immatura } \\
\hline \multirow{2}{*}{\multicolumn{2}{|c|}{$\begin{array}{l}\text { Sphaeroidinellopsis seminulina } \\
\text { S. subdehiscens }\end{array}$}} & 1 & & 1 & & & & & \\
\hline & & & & & & & & & \\
\hline \multicolumn{2}{|c|}{ Indeterminants (juveniles) } & & & & $\begin{array}{llll}1 & 2 & 1 & 1\end{array}$ & $\begin{array}{ll}1 & 1 \\
\end{array}$ & 1 & 3 & 1 \\
\hline
\end{tabular}

apleistocene 


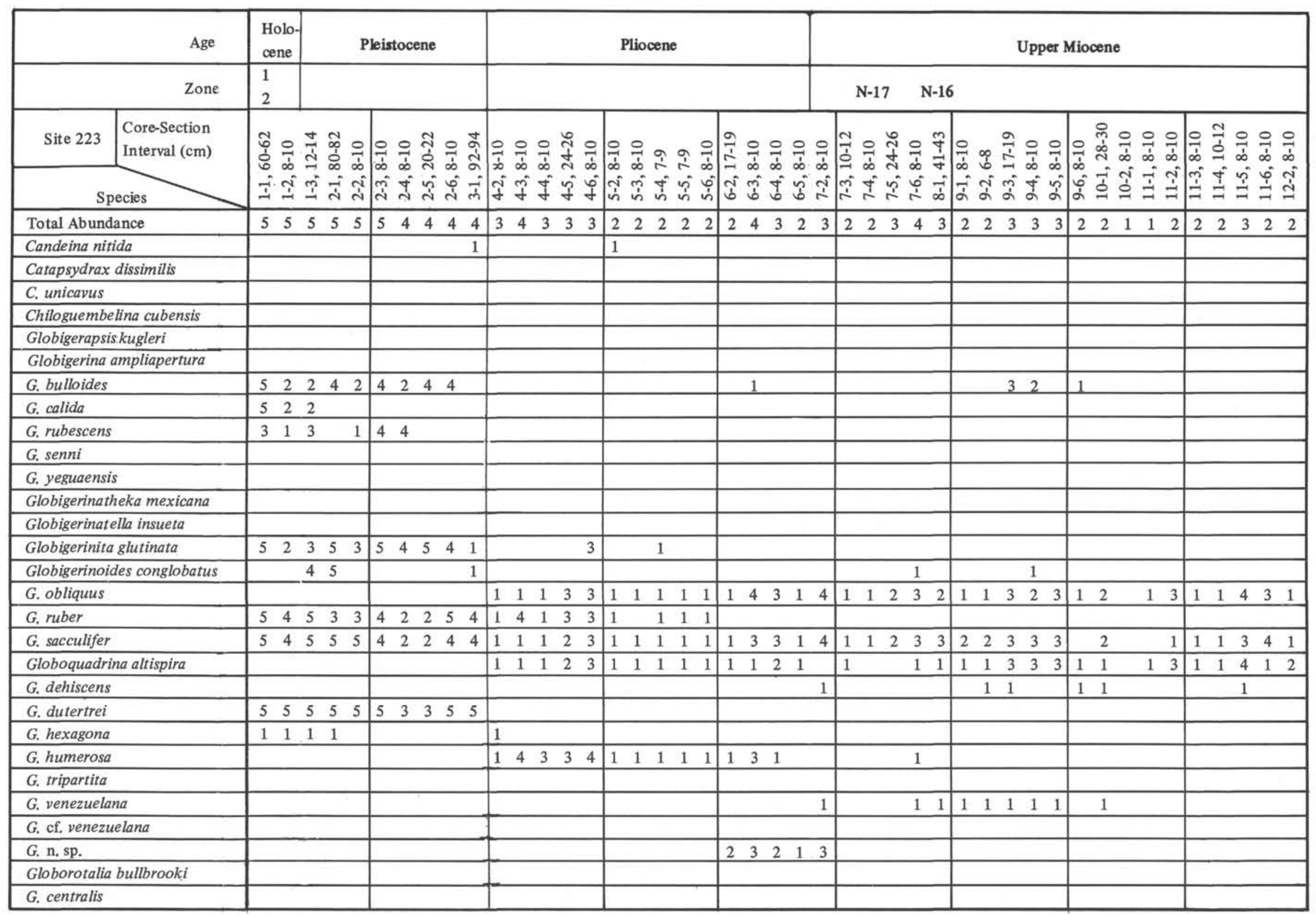




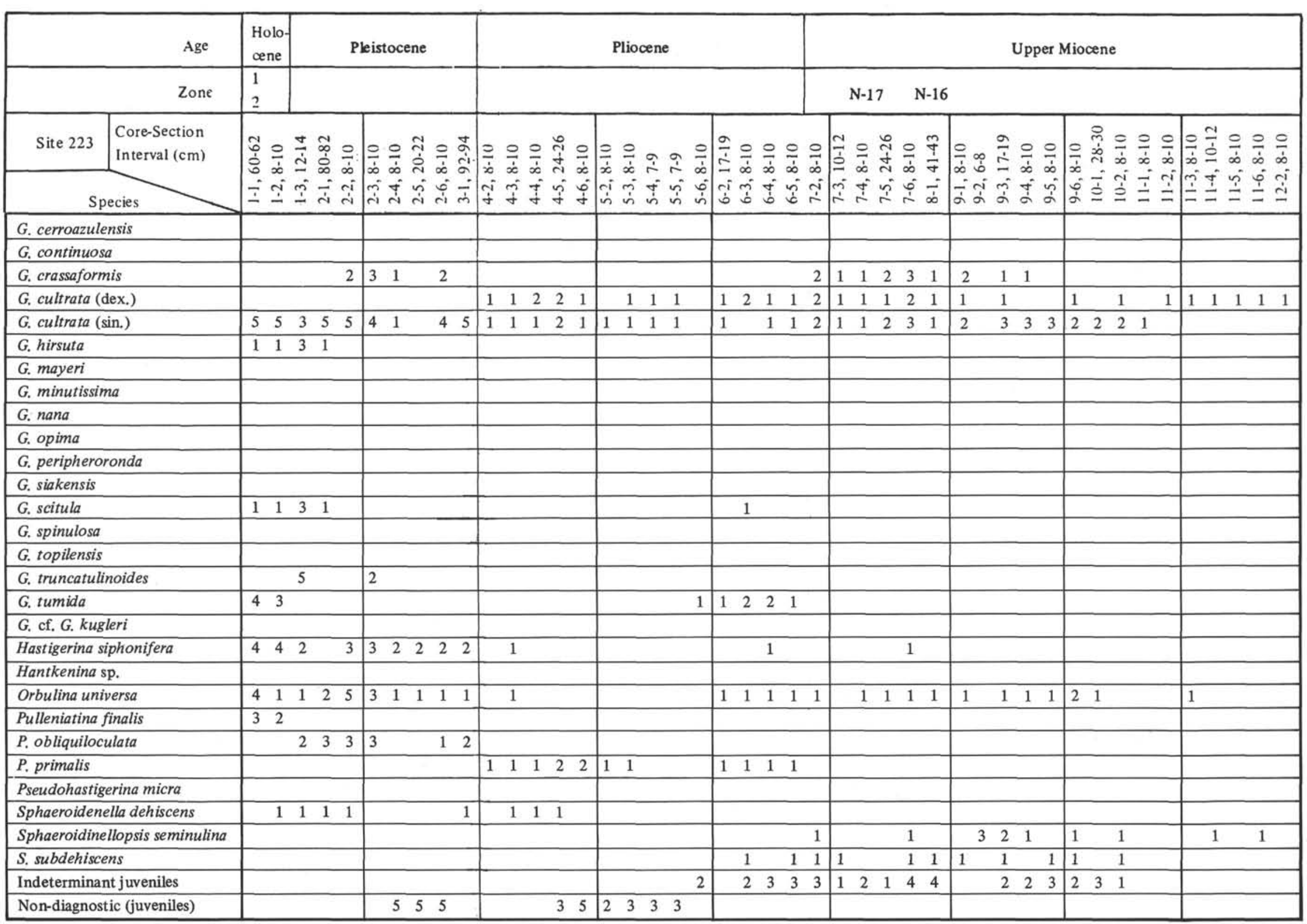




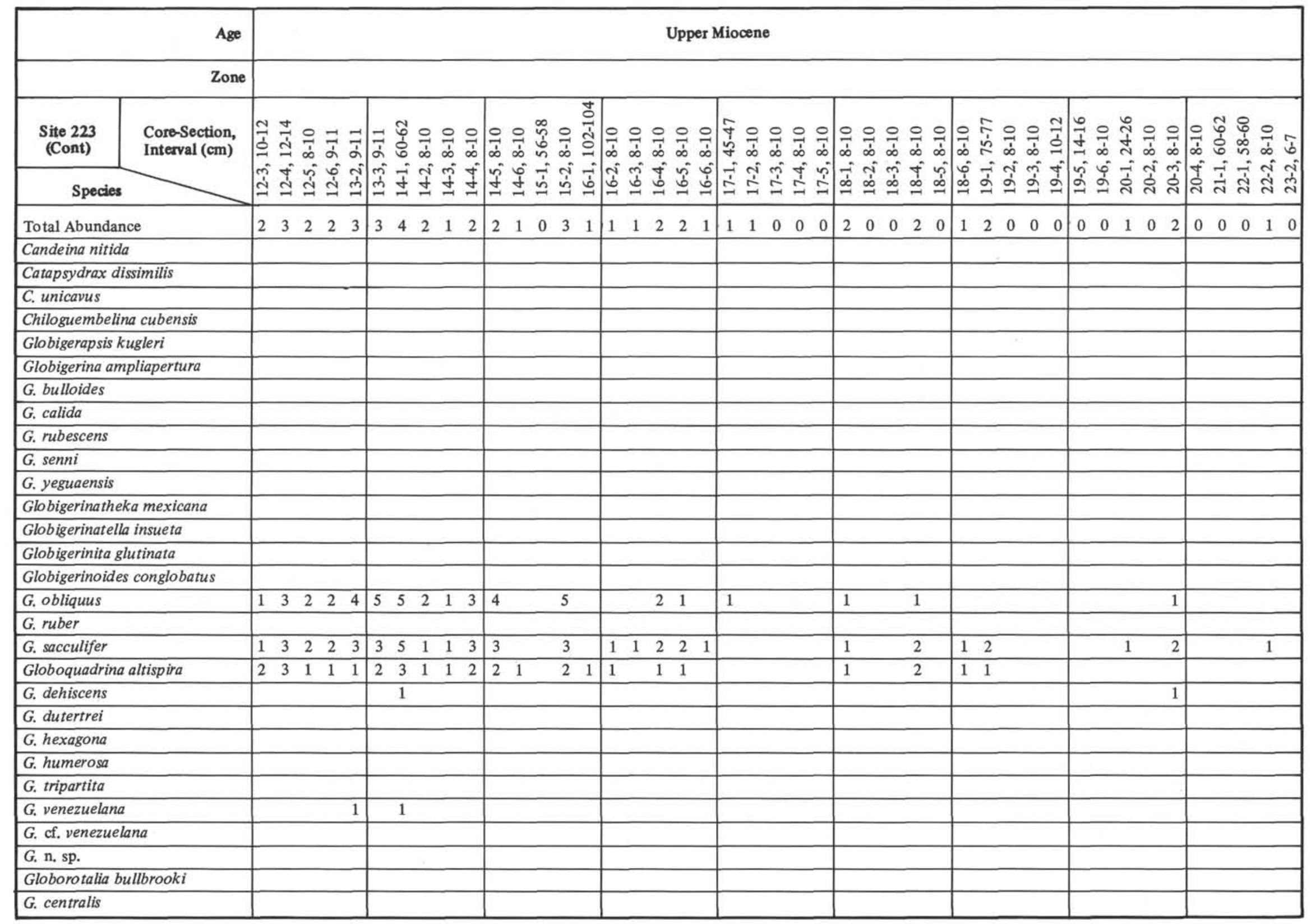




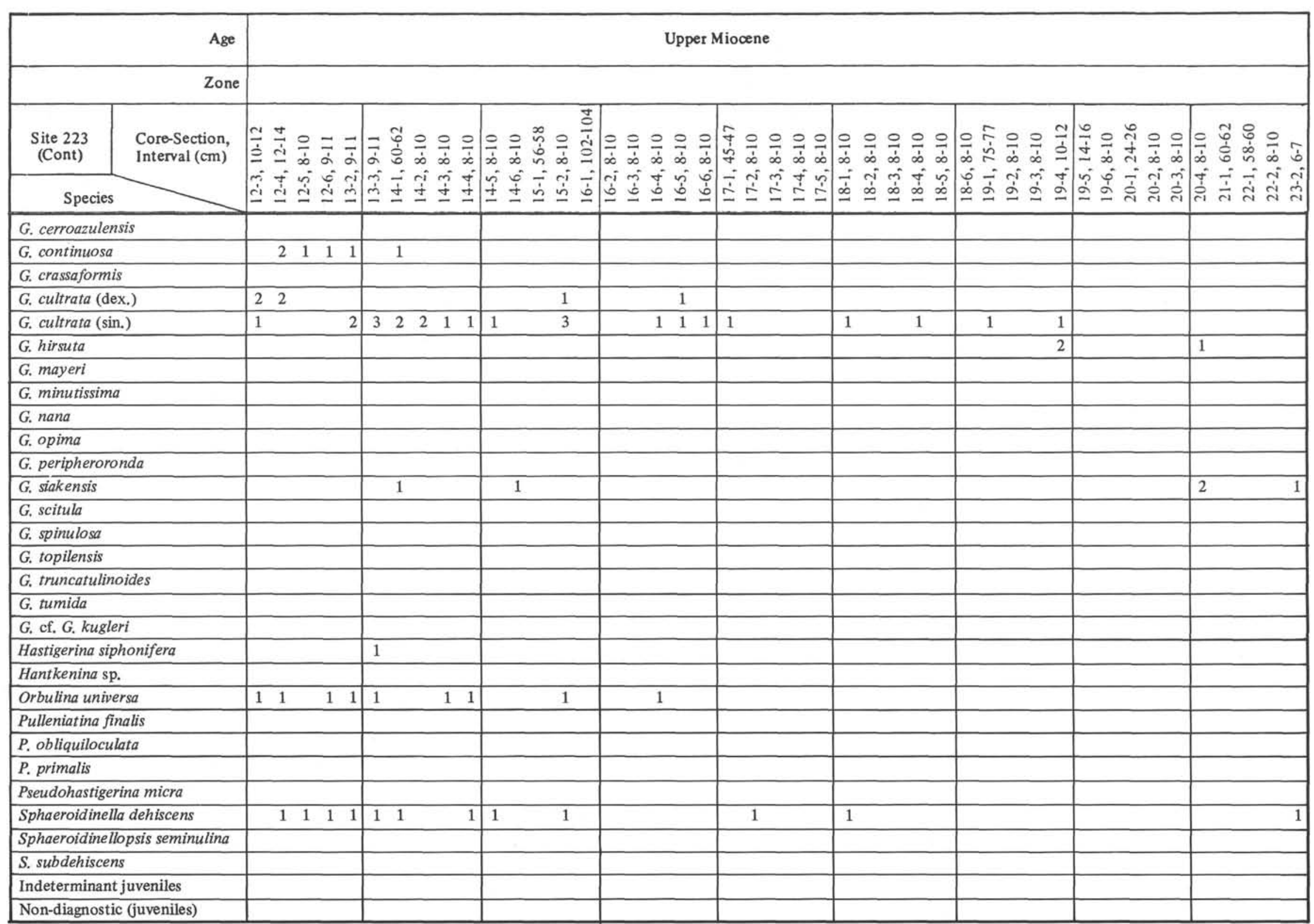




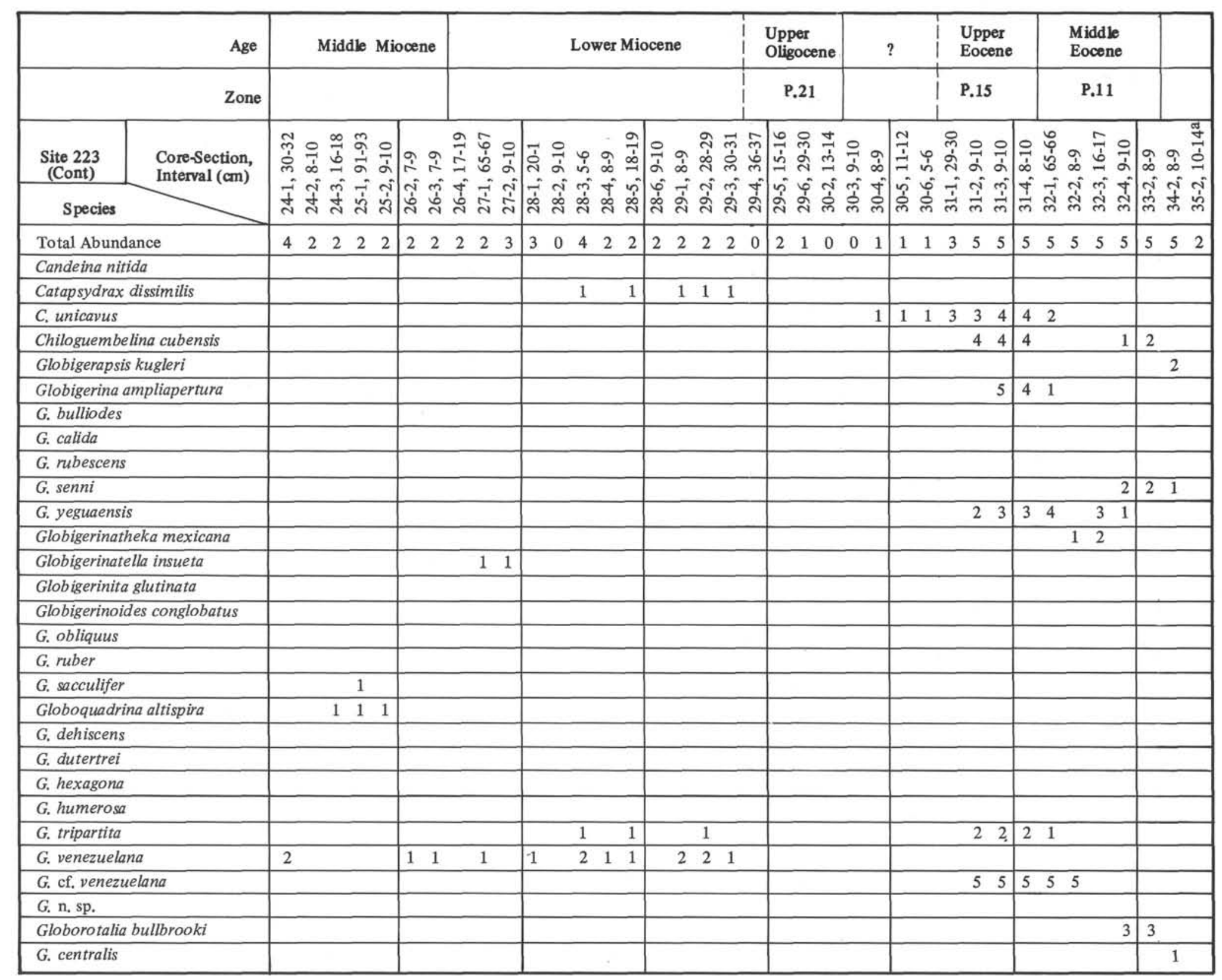




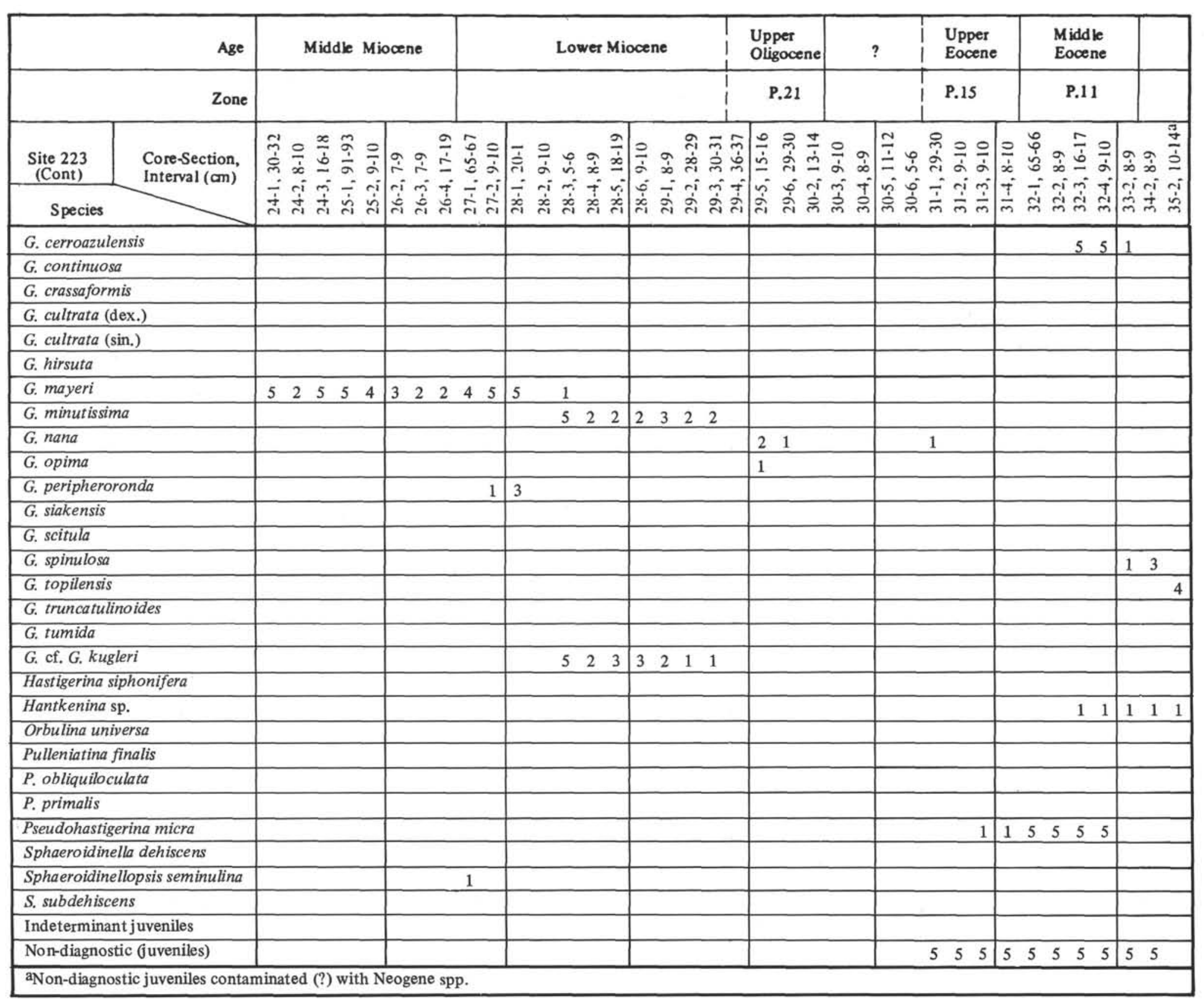




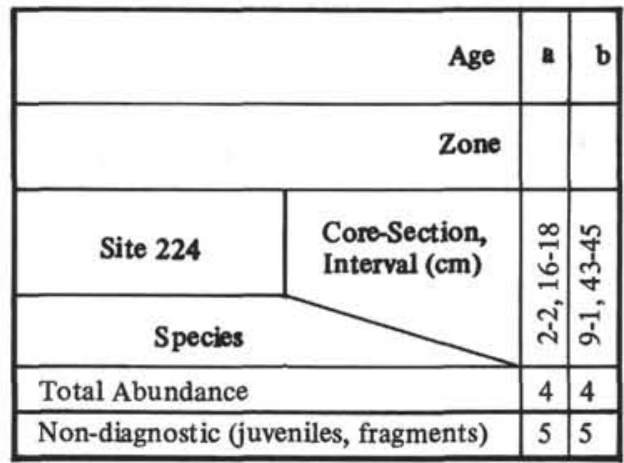

aUpper Miocene

bLower Oligocene 


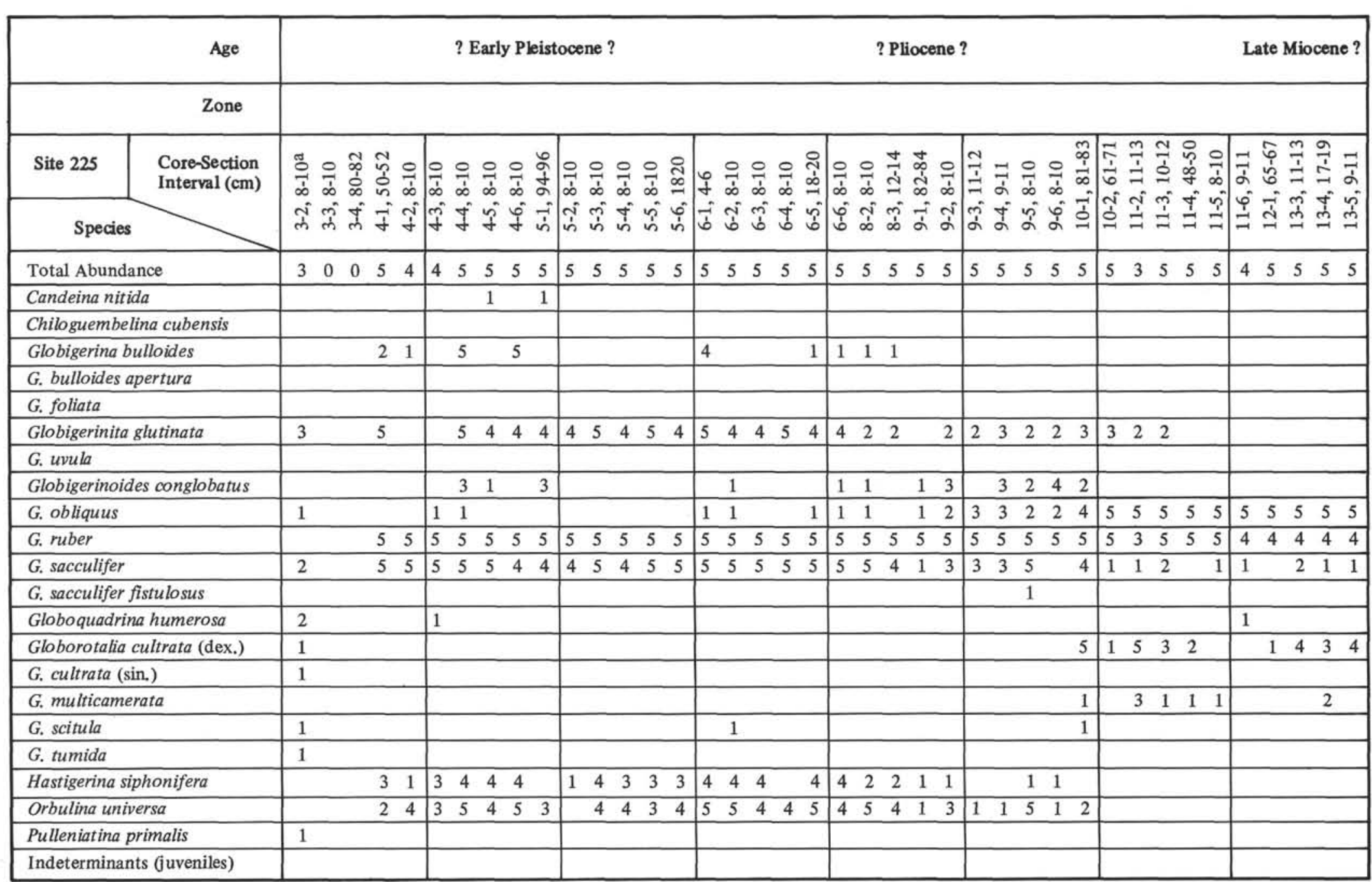




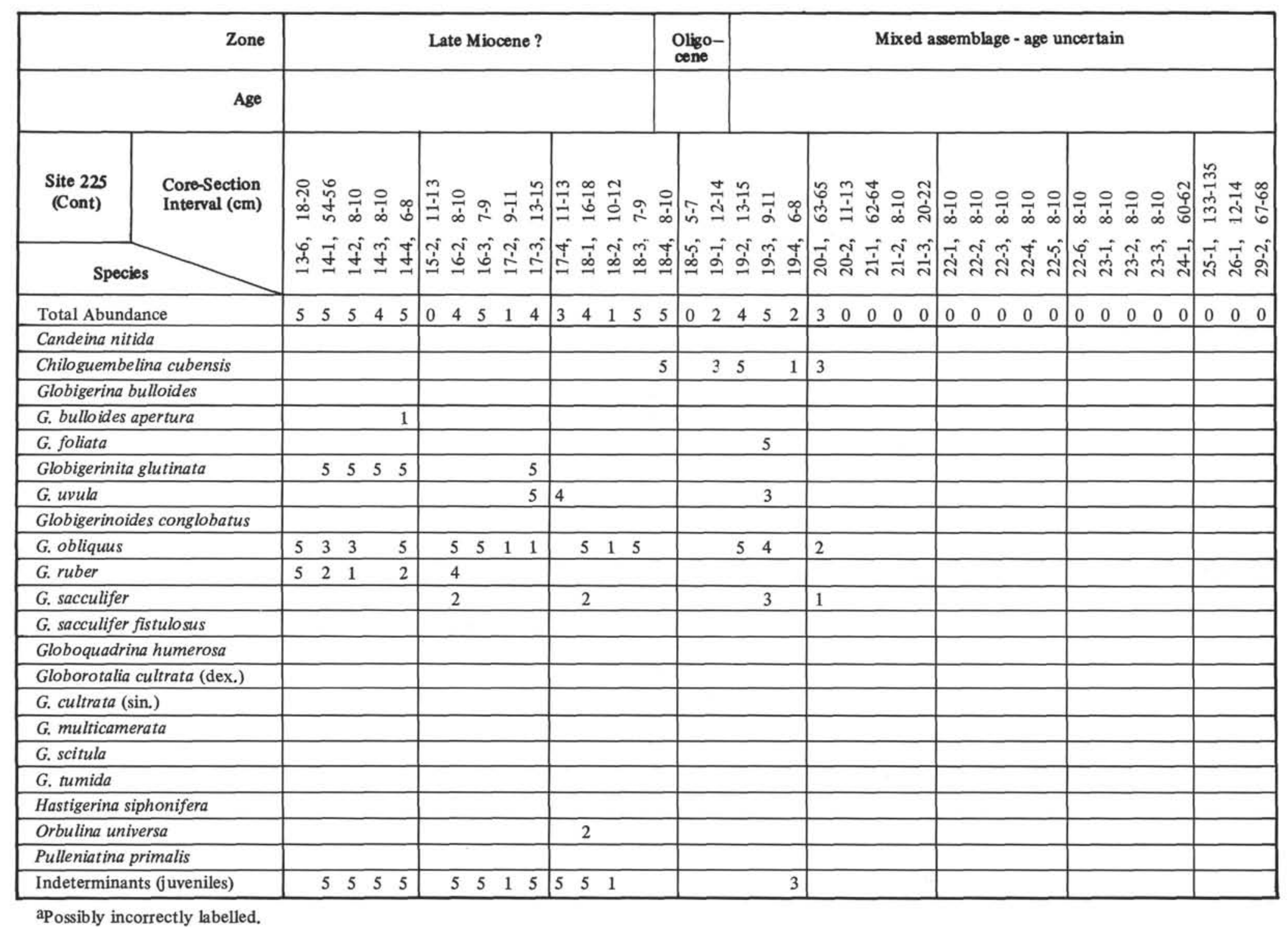




\begin{tabular}{|c|c|c|c|}
\hline & Age & \multicolumn{2}{|c|}{$\mathrm{Q}$ ? } \\
\hline & Zone & \multicolumn{2}{|c|}{$23 ?$} \\
\hline Site 226 & $\begin{array}{l}\text { Core-Section, } \\
\text { Interval }(\mathrm{cm})\end{array}$ & $\begin{array}{l}0 \\
\exists \\
\dot{J} \\
\Xi\end{array}$ & $\frac{\circ}{\infty}$ \\
\hline \multicolumn{2}{|c|}{ Species } & $\stackrel{?}{-1}$ & $\stackrel{8}{8}$ \\
\hline \multicolumn{2}{|c|}{ Total Abundance } & 1 & 2 \\
\hline \multicolumn{2}{|c|}{ Globigerina rubescens } & & 1 \\
\hline \multicolumn{2}{|c|}{ Globigerinita glutinata } & & 1 \\
\hline \multicolumn{2}{|c|}{ Globigerinoides ruber } & & 1 \\
\hline \multicolumn{2}{|c|}{ G. sacculifer } & & 1 \\
\hline \multicolumn{2}{|c|}{ Hastigerina siphonifera } & 1 & 1 \\
\hline
\end{tabular}




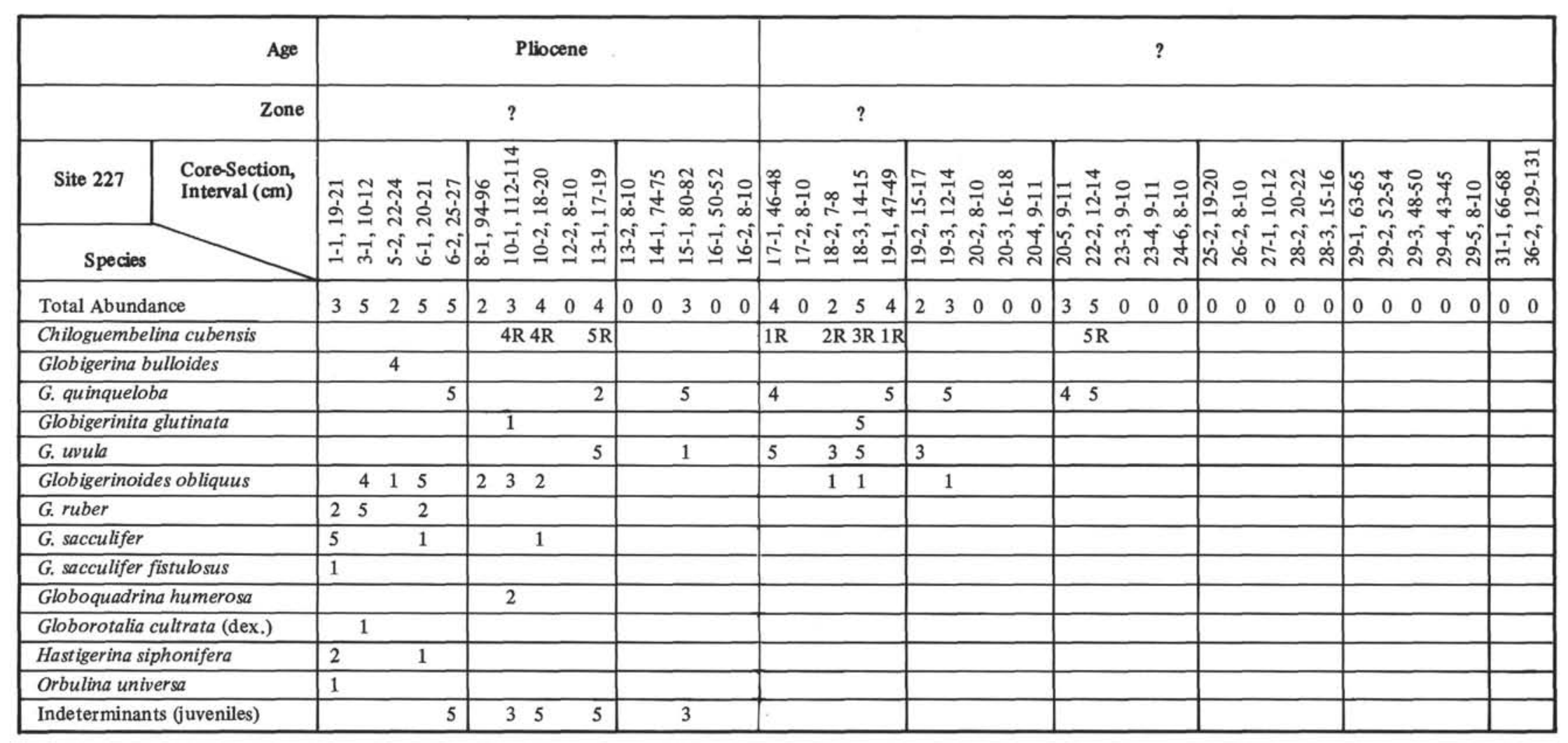




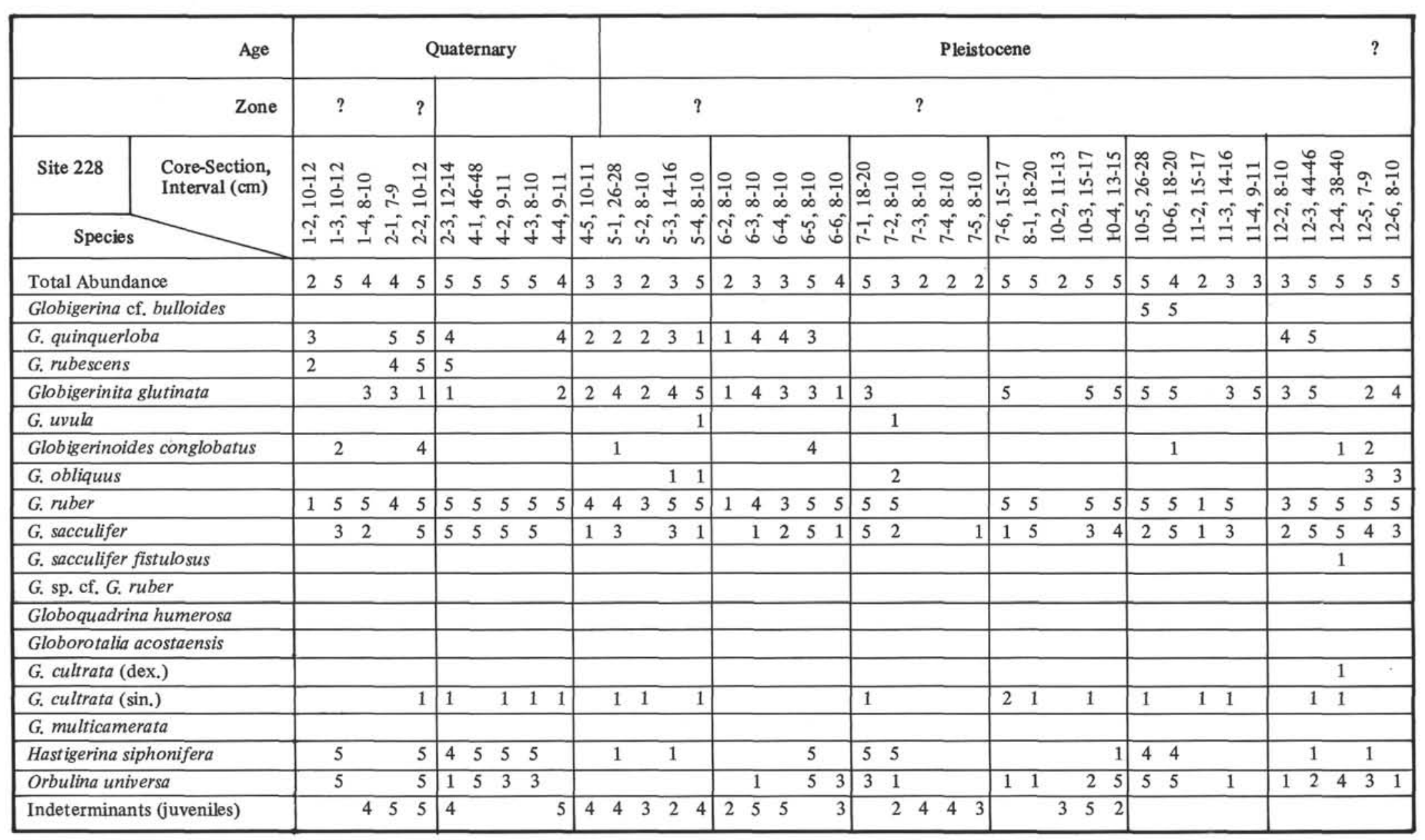




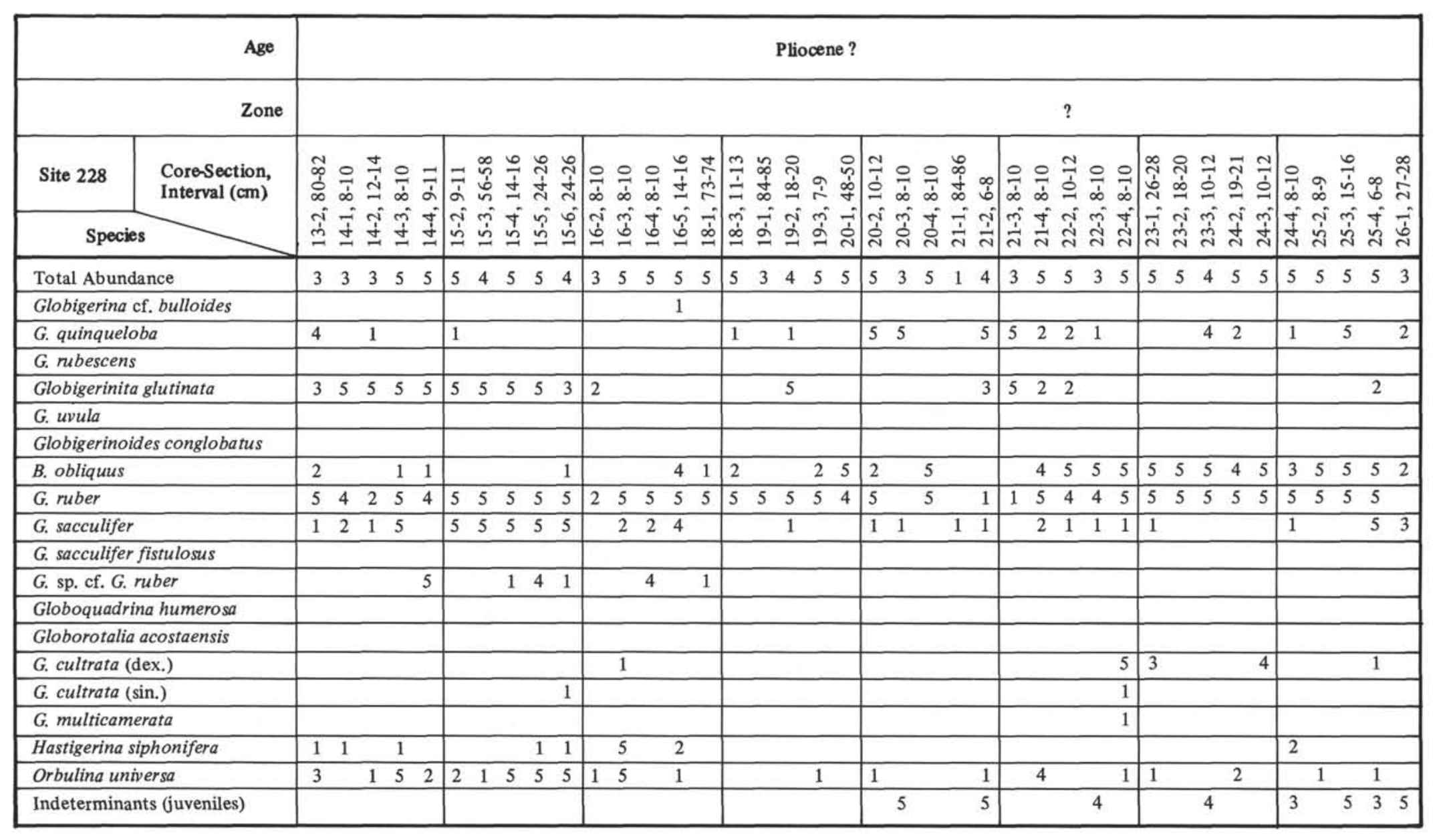




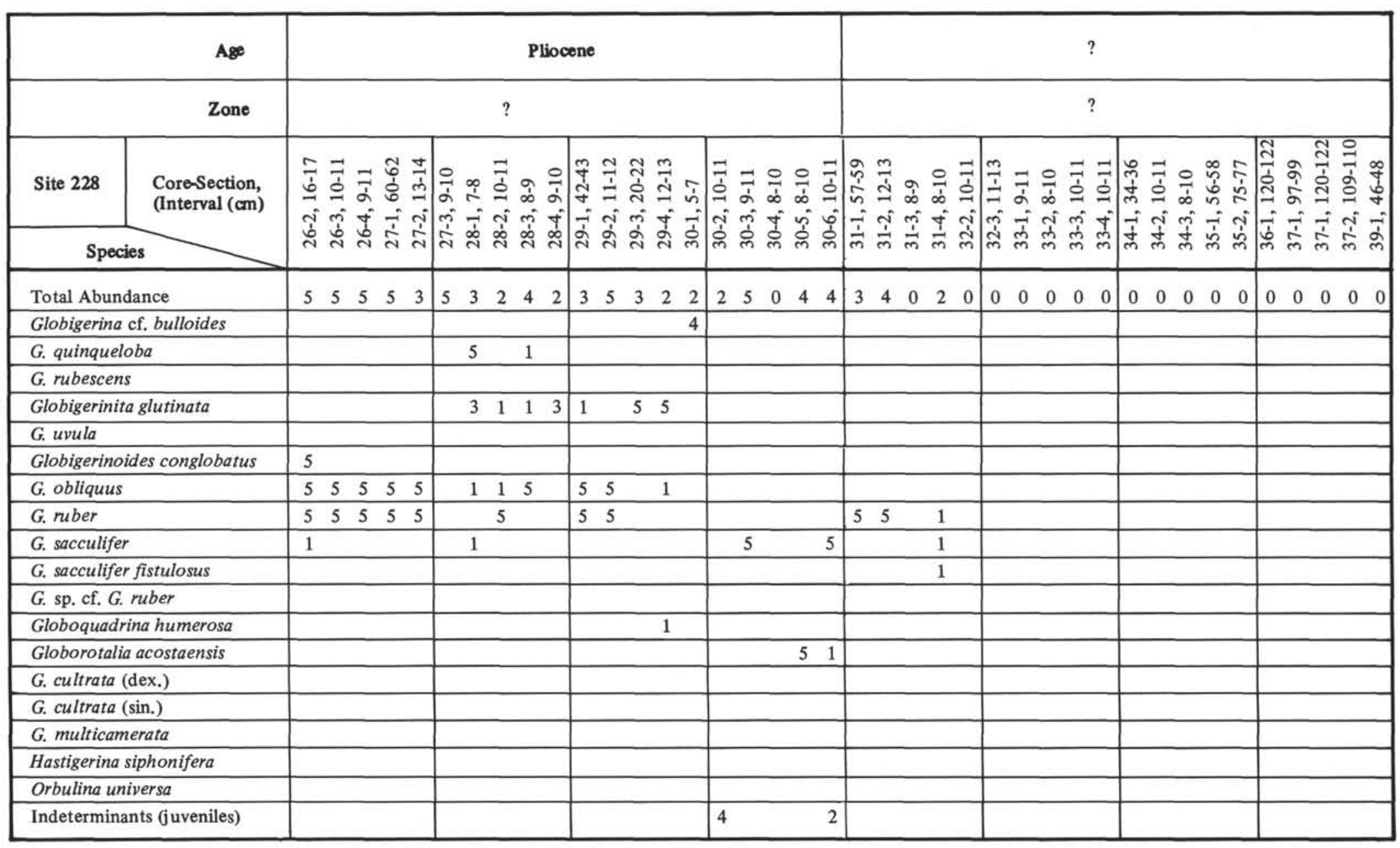




\begin{tabular}{|c|c|c|c|c|c|c|c|c|c|c|c|c|c|c|c|c|c|c|c|c|}
\hline & Age & \multicolumn{19}{|c|}{ Quaternary } \\
\hline & Zone & \multicolumn{5}{|c|}{ ? } & \multicolumn{9}{|c|}{ ? } & \multicolumn{5}{|c|}{ ? } \\
\hline Hole 229 & $\begin{array}{l}\text { Core-Section, } \\
\text { Interval (cm) }\end{array}$ & 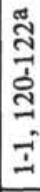 & $\begin{array}{l}\stackrel{\circ}{1} \\
\dot{0} \\
\vec{a}\end{array}$ & & $\begin{array}{l}0 \\
\dot{\infty} \\
\dot{n} \\
\dot{n}\end{array}$ & $\begin{array}{l} \pm \\
\dot{a} \\
- \\
\pm \\
\Delta\end{array}$ & $\begin{array}{l}\text { - } \\
\infty \\
\text { ஸे }\end{array}$ & & $\begin{array}{l}\frac{m}{\leftrightarrows} \\
\dot{\vec{n}}\end{array}$ & $\begin{array}{l}\dot{a} \\
\text { ஸे } \\
\dot{n}\end{array}$ & $\begin{array}{l}\infty \\
\vdots \\
0 \\
n \\
n \\
n\end{array}$ & $\begin{array}{l}0 \\
\infty \\
\infty \\
\dot{y} \\
\text { m. }\end{array}$ & $\begin{array}{l}0 \\
\dot{y} \\
\tilde{c} \\
\text { mं }\end{array}$ & 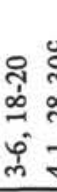 & 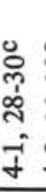 & 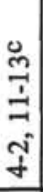 & $\begin{array}{l}\overrightarrow{1} \\
\stackrel{1}{1} \\
\tilde{y}\end{array}$ & 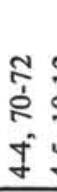 & & $\begin{array}{l}\circ \\
\infty \\
0 \\
+\end{array}$ \\
\hline \multicolumn{2}{|c|}{ Total Abundance } & 3 & 3 & 5 & 5 & 5 & 5 & 5 & 4 & 5 & 3 & 5 & 55 & $5 ?$ & 3 & 3 & 4 & 3 & 3 & 3 \\
\hline \multicolumn{2}{|c|}{ Globigerina bulloides } & 5 & 2 & 5 & 5 & 5 & 5 & 5 & & 5 & 3 & 5 & 5 & 4 & 1 & 1 & 1 & & & \\
\hline \multicolumn{2}{|c|}{ G. quinqueloba } & & & & & & & & 5 & 5 & 3 & 2 & & & & & & 4 & 2 & 2 \\
\hline \multicolumn{2}{|c|}{ Globigerinita glutinata } & 4 & 5 & 4 & 3 & 2 & 5 & 1 & 3 & 5 & 4 & 5 & 32 & 4 & 1 & & 3 & 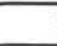 & 1 & \\
\hline \multicolumn{2}{|c|}{ Globigerinoides ruber } & 5 & 1 & 5 & 5 & 5 & 5 & 5 & 1 & 5 & & 5 & 55 & 5 & 4 & & 5 & 3 & 3 & 3 \\
\hline \multicolumn{2}{|c|}{ G. sacculifer } & 5 & & 5 & 5 & & 5 & & & & & & 21 & 11 & 1 & & 1 & & & \\
\hline \multicolumn{2}{|c|}{ Globoquadrina dutertrei } & 1 & & 2 & 1 & & 2 & 1 & & & & & 1 & & & 1 & 1 & & & \\
\hline \multicolumn{2}{|c|}{ Globorotalia crassaformis } & & & & & & & & & & & & 11 & 1 & 1 & 1 & 1 & & & \\
\hline \multicolumn{2}{|c|}{ G. cultrata (dex.) } & & & & & & 1 & & & & & & & & & & & & & \\
\hline \multicolumn{2}{|c|}{ G. cultrata (sin.) } & 1 & & 2 & 3 & 3 & 2 & 1 & & 1 & 1 & 4 & 34 & 42 & 2 & 3 & 4 & 1 & 1 & 1 \\
\hline \multicolumn{2}{|c|}{ G. hirsuta } & 1 & & 1 & & & 1 & 1 & & & & 1 & & & & & & & & \\
\hline \multicolumn{2}{|c|}{ G. truncatulinoides } & & & & & & & & & & & & & & & 1 & & & & \\
\hline \multicolumn{2}{|c|}{ Hastigerina siphonifera } & 4 & & 5 & 5 & 5 & 4 & & & 2 & 2 & 5 & 15 & 52 & 2 & 3 & 4 & 1 & 1 & 1 \\
\hline \multicolumn{2}{|c|}{ Orbulina universa } & 5 & & 5 & 4 & 4 & 2 & 1 & & 1 & 1 & 2 & 13 & 31 & 1 & 4 & 1 & 1 & 1 & 1 \\
\hline \multicolumn{2}{|c|}{ Pulleniatina obliquiloculata } & & & 1 & & & 1 & & & & & & 11 & 1 & & & & & & \\
\hline \multicolumn{2}{|c|}{ Indeterminants (juveniles) } & & & & & & & & & & & & & & & & & 5 & 5 & \\
\hline
\end{tabular}

aMostly pteropods

bSmall pteropods, juvenile forams, rads

Mainly otoliths, worm tuses, and echinoid fragments. Also large streblus beccarii, pteropods 


\begin{tabular}{|c|c|c|c|c|c|c|c|c|c|c|c|c|c|c|c|c|c|c|c|c|c|c|c|c|c|c|c|c|c|c|}
\hline & Age & \multicolumn{29}{|c|}{ Quaternary } \\
\hline & Zone & \multicolumn{29}{|c|}{ ? } \\
\hline Hole 229A & $\begin{array}{l}\text { Core-Section, } \\
\text { Interval }(\mathrm{cm})\end{array}$ & \multicolumn{5}{|c|}{ 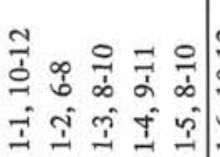 } & \multicolumn{3}{|c|}{ 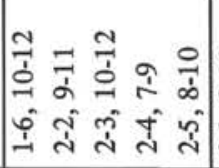 } & \multicolumn{4}{|c|}{ 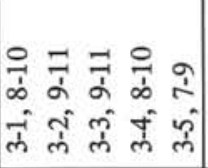 } & \multicolumn{5}{|c|}{ 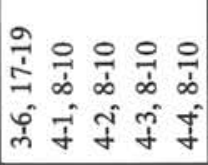 } & \multicolumn{4}{|c|}{ 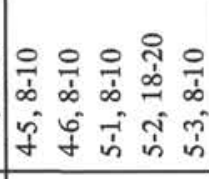 } & \multicolumn{2}{|c|}{ 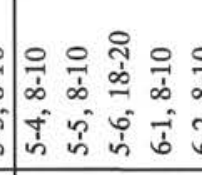 } & \multicolumn{2}{|c|}{ 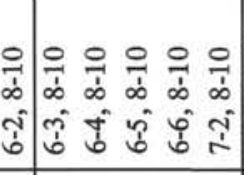 } & \multicolumn{4}{|c|}{ 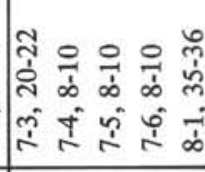 } \\
\hline \multirow{2}{*}{\multicolumn{2}{|c|}{$\begin{array}{l}\text { Total Abundance } \\
\text { Globigerina bulloides }\end{array}$}} & 5 & 3 & 3 & 5 & & & 35 & $4 \quad 3$ & 5 & 5 & 4 & $5 \quad 4$ & & s & 5 & 5 : & 5 & 5 & 55 & 5 & & 34 & 35 & 4 & $\begin{array}{lllll}4 & 5 & 5 & 5 & 5\end{array}$ & & 3 & 4 & 42 \\
\hline & & & & 2 & 5 & & & $5 \quad 5$ & $5 \quad 5$ & 5 & 5 & 5 & $5 \quad 5$ & & 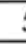 & 5 & 55 & 5 & 5 & 54 & 5 & & $\begin{array}{ll}1 & 1 \\
\end{array}$ & 25 & \begin{tabular}{l|l}
5 & 1 \\
\end{tabular} & \begin{tabular}{lllll|}
1 & 2 & 5 & 5 & 5 \\
\end{tabular} & & 2 & 45 & $5 \quad 2$ \\
\hline \multicolumn{2}{|c|}{ G. quinqueloba } & 5 & 5 & 4 & 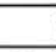 & & & 32 & 23 & & 1 & & & & $s$ & 5 & 43 & 3 & 2 & 35 & 3 & & 55 & $5 \quad 5$ & \begin{tabular}{l|l}
5 & 5 \\
5
\end{tabular} & $\begin{array}{|lllll|}5 & 5 & 4 & 5 & 5 \\
\end{array}$ & & 5 & 3 & \\
\hline \multicolumn{2}{|c|}{ Globigerinita glutinata } & 5 & 4 & 5 & 5 & & & $4 \quad 3$ & 34 & 4 & 3 & 4 & 2 & & 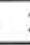 & 4 & 5 & 3 & & 44 & 4 & 1 & 13 & $\begin{array}{ll}3 & 3 \\
\end{array}$ & 3 : & \begin{tabular}{lllll|}
5 & 5 & 5 & 5 & 5 \\
\end{tabular} & & 5 & 55 & $\begin{array}{ll}5 & 1 \\
\end{array}$ \\
\hline \multicolumn{2}{|c|}{ G. uvula } & 2 & & & & & & & & & & 1 & & & & & & & & & & & 1 & & & 1 & & & & \\
\hline \multicolumn{2}{|c|}{ Globigerinoides conglobatus } & & & & & 1 & & & & 1 & 1 & & 1 & L. & & & 11 & & 1 & & & 1 & & & \begin{tabular}{l|l}
1 \\
\end{tabular} & & & & & \\
\hline \multicolumn{2}{|c|}{ G. ruber } & 4 & & 2 & 5 & & & $5 \quad 5$ & $\begin{array}{ll}5 & 4\end{array}$ & 5 & 4 & 5 & 53 & & 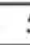 & 5 & 5 & 5 & 5 & 55 & 5 & \begin{tabular}{l|l}
5 \\
\end{tabular} & $\begin{array}{lll}22 \\
\end{array}$ & 25 & \begin{tabular}{l|l}
5 & 2 \\
\end{tabular} & \begin{tabular}{lllll|}
2 & 2 & 5 & 5 & 5 \\
\end{tabular} & & 4 & 55 & $\begin{array}{ll}5 & 2 \\
\end{array}$ \\
\hline \multicolumn{2}{|l|}{ G. sacculifer } & & & & 2 & & & 1 & $\begin{array}{ll}1 & 1 \\
\end{array}$ & 1 & 3 & 4 & 11 & & 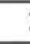 & 3 & 35 & 5 . & 5 & 53 & 3 & \begin{tabular}{l|l}
5 & $1-10$ \\
\end{tabular} & \begin{tabular}{ll|}
1 & 1 \\
\end{tabular} & 24 & \begin{tabular}{l|l}
5 & \\
\end{tabular} & \begin{tabular}{llll|}
1 & 5 & 5 & 5 \\
\end{tabular} & & 1 & 4 & 3 \\
\hline \multirow{2}{*}{\multicolumn{2}{|c|}{$\begin{array}{l}\text { Globoquadrina dutertrei } \\
\text { Globorotalia crassaformis }\end{array}$}} & & & & 1 & & & & 1 & & 1 & 1 & & & & 1 & 31 & 1 & 4 & 32 & 3 & \begin{tabular}{l|l}
3 & 1 \\
\end{tabular} & $\begin{array}{ll}1 & 1 \\
\end{array}$ & 12 & 2 & \begin{tabular}{lllll|}
1 & 1 & 3 & 5 & 4 \\
\end{tabular} & & 1 & 1 & 1 \\
\hline & & & & & & & & & 1 & & & 1 & & & & & 1 & & & & & & & & & $\begin{array}{ll} & 1\end{array}$ & & 1 & 11 & $\begin{array}{ll}1 & 1\end{array}$ \\
\hline \multicolumn{2}{|c|}{ G. cultrata (dex.) } & & & & & & & & & & & 1 & & & & & 1 & & & & & & & & & & & & & \\
\hline \multicolumn{2}{|c|}{ G. cultrata $(\sin )}$, & & & 2 & & & & 2 & 11 & 1 & & 2 & 1 & & 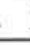 & 2 & 2 & & 4 & 22 & 4 & 4 & 1 & 3 & 4. & \begin{tabular}{lllll|}
1 & 1 & 3 & 4 & 4 \\
\end{tabular} & & 1 & 2 & 1 \\
\hline \multirow{2}{*}{\multicolumn{2}{|c|}{$\frac{\text { G. hirsuta }}{\text { G. scitula }}$}} & & & & & & & & & & & & & & & & & & & & & & & & & & & & & \\
\hline & & & & & & & & & & & & & & & & & & & & & & & & & & 1 & & 1 & & \\
\hline \multicolumn{2}{|c|}{ Hastigerina siphonifera } & 5 & & & 3 & & & $\begin{array}{ll}1 & 1 \\
\end{array}$ & 21 & 2 & 4 & 5 & $\begin{array}{ll}3 & 4 \\
\end{array}$ & & 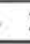 & 3 & 2 & 3 | & 3 & 32 & 23 & 3 & 1 & 12 & 3 & \begin{tabular}{lll|}
3 & 4 & 4 \\
\end{tabular} & & & & 21 \\
\hline Orbulina uni & ersa & & 1 & & 4 & & & 1 & 1 & 5 & 4 & 4 & 22 & & 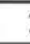 & 1 & 2 & 5 & 5 & 42 & 4 & \begin{tabular}{l|l}
5 \\
\end{tabular} & \begin{tabular}{ll|}
1 & 1 \\
\end{tabular} & 2 & 4 & \begin{tabular}{lll|}
4 & 5 & 4 \\
\end{tabular} & & & 1 & 1 \\
\hline Pulleniatina & bliquiloculata & & & & & & & & 1 & & & & & & & & & & & & & & & & 1 & & & 1 & & \\
\hline Indetermina & ts (juveniles) & 5 & 5 & 5 & & & & & & & & & & & & & & & & & & & & 5 & & $\begin{array}{ll}5 & 5\end{array}$ & & & & \\
\hline
\end{tabular}




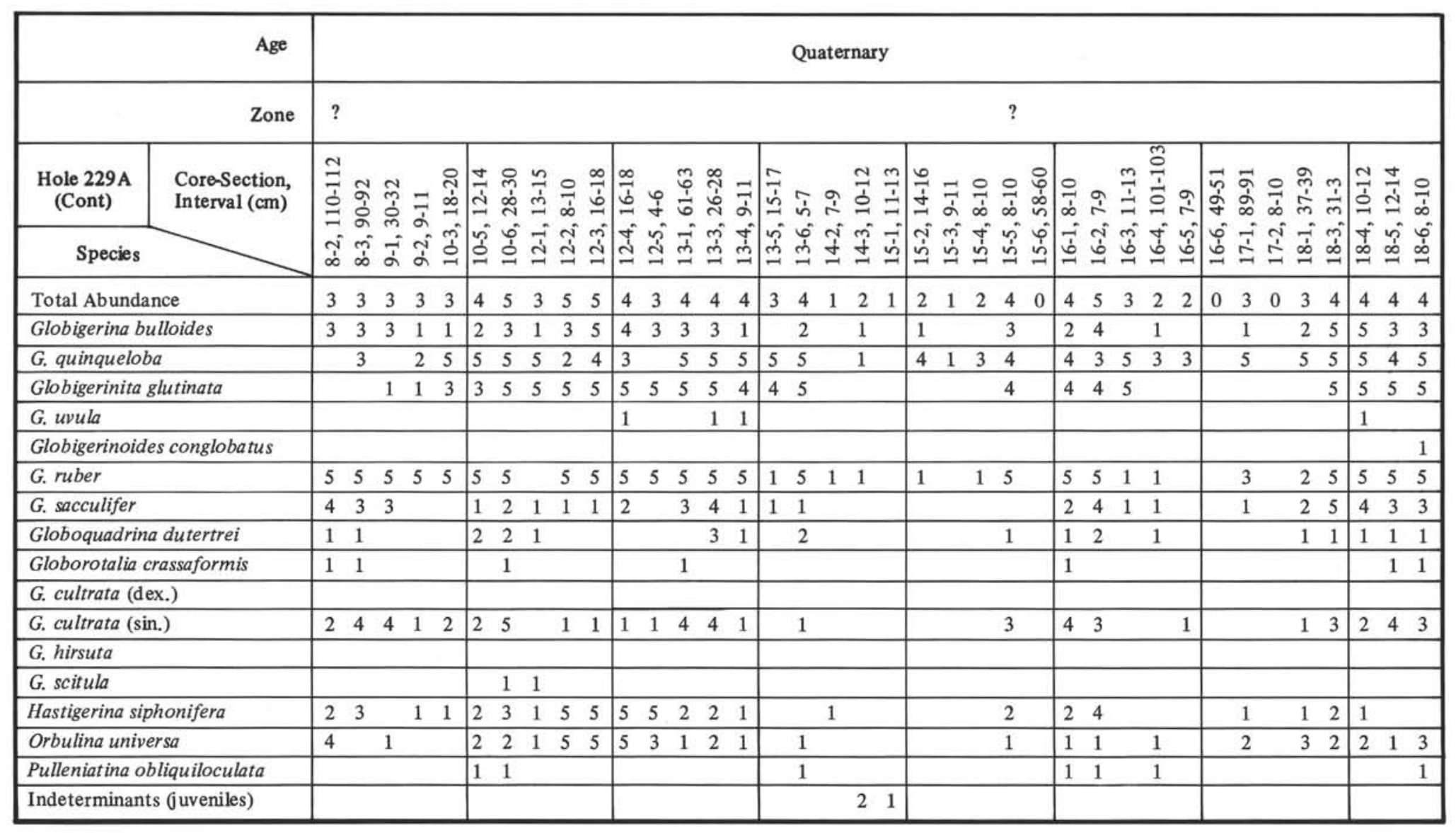




\begin{tabular}{|c|c|c|c|c|}
\hline & Age & \multicolumn{3}{|c|}{$\begin{array}{l}\text { Quater- } \\
\text { nary }\end{array}$} \\
\hline \multicolumn{2}{|r|}{ Zone } & \multicolumn{3}{|c|}{$?$} \\
\hline Site 230 & $\begin{array}{l}\text { Core-Section, } \\
\text { Interval }(\mathrm{cm})\end{array}$ & \multirow{2}{*}{\multicolumn{3}{|c|}{ 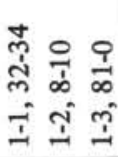 }} \\
\hline \multicolumn{2}{|c|}{ Species } & & & \\
\hline \multicolumn{2}{|c|}{ Total Abundance } & & 3 & 5 \\
\hline \multicolumn{2}{|c|}{ Globigerina bulloides } & 2 & 4 & 5 \\
\hline \multicolumn{2}{|c|}{ G. quinqueloba } & 4 & & 3 \\
\hline \multicolumn{2}{|c|}{ Globigerinita ghutinata } & & 5 & 4 \\
\hline \multicolumn{2}{|c|}{ G. uvula } & & & 1 \\
\hline \multicolumn{2}{|c|}{ Globigerinoides ruber } & & 5 & 5 \\
\hline \multicolumn{2}{|c|}{ G. sacculifer } & & 3 & 2 \\
\hline \multicolumn{2}{|c|}{ Globoquadrina dutertrei } & & & 1 \\
\hline \multicolumn{2}{|c|}{ Globorotalia crassaformis } & \multicolumn{3}{|c|}{1} \\
\hline \multicolumn{2}{|c|}{ G. cultrata $(\sin )}$. & 1 & 1 & \\
\hline \multicolumn{2}{|c|}{ Hastigerina siphonifera } & & 5 & 5 \\
\hline \multicolumn{2}{|c|}{ Orbulina universa } & 2 & 1 & 3 \\
\hline
\end{tabular}

